\title{
Large-Scale and Flexible Optical Synapses for Neuromorphic Computing and Integrated Visible Information Sensing-Memory-Processing
}

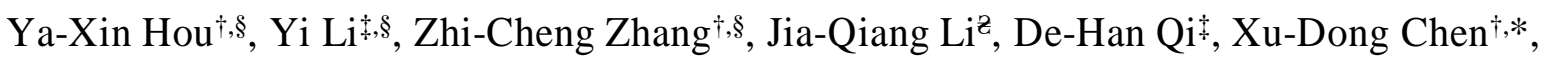
Jing-Jing Wang ${ }^{\dagger}$, Bin-Wei Yao ${ }^{\dagger}$, Mei-Xi Yu', Tong-Bu Lü,*, and Jin Zhang,**

${ }^{\dagger} \mathrm{MOE}$ International Joint Laboratory of Materials Microstructure, Institute for New Energy Materials and Low Carbon Technologies, School of Material Science and Engineering, and łKey Laboratory of Computer Vision and Systems (Ministry of Education), School of Computer Science and Engineering, Tianjin University of Technology, Tianjin 300384, China ${ }^{z}$ Center for Nanochemistry, Beijing Science and Engineering Center for Nanocarbons, Beijing National Laboratory for Molecular Sciences, College of Chemistry and Molecular Engineering, Peking University, Beijing 100871, China

$\S$ These authors contributed equally to this work.

*Corresponding author. Xu-Dong Chen, chenxd@email.tjut.edu.cn; Tong-Bu Lu, lutongbu@tjut.edu.cn; Jin Zhang, jinzhang@pku.edu.cn 
Table S1. Comparisons of optoelectronic and fully light-modulated synapses based on 2D materials and other material systems.

\begin{tabular}{|c|c|c|c|c|c|c|c|c|c|c|c|}
\hline \multirow{2}{*}{ Active layer } & \multirow{2}{*}{$\begin{array}{l}\text { Three/two } \\
\text { terminals }\end{array}$} & \multicolumn{2}{|c|}{ Operation mode } & \multicolumn{2}{|c|}{ Nonlinearity } & \multirow{2}{*}{$\mathrm{CCV}$} & \multirow{2}{*}{$\mathrm{DDV}$} & \multicolumn{3}{|c|}{ ANN } & \multirow{2}{*}{ Ref. } \\
\hline & & LTP & LTD & $\beta \mathrm{P}$ & $\beta_{\mathrm{D}}$ & & & Network & Accuracy & Epochs & \\
\hline $\begin{array}{c}\text { Pyr-GDY/ } \\
\text { Gr/PbS-QDs }\end{array}$ & Two & $980 \mathrm{~nm}$ & $450 \mathrm{~nm}$ & 1.7 & 1.9 & $2.8 \%$ & $<4 \%$ & $784 \times 300 \times 10$ & $90.8 \%$ & 40 & $\begin{array}{l}\text { This } \\
\text { work }\end{array}$ \\
\hline $\mathrm{BP}$ & Two & $280 \mathrm{~nm}$ & $365 \mathrm{~nm}$ & 1 & 1 & - & - & $784 \times 10$ & $90 \%$ & 1000 & 1 \\
\hline $\mathrm{ZnO} / \mathrm{PbS}$ & Two & $365 \mathrm{~nm}$ & $980 \mathrm{~nm}$ & - & - & - & - & $785 \times 10$ & 67 & 4000 & 2 \\
\hline $\mathrm{Bi}_{2} \mathrm{O}_{2} \mathrm{Se} / \mathrm{Gr}$ & Three & $635 \mathrm{~nm}$ & $365 \mathrm{~nm}$ & - & - & - & - & - & - & - & 3 \\
\hline $\mathrm{MoO}_{\mathrm{x}}$ & Two & $365 \mathrm{~nm}$ & - & - & - & - & - & - & - & - & 4 \\
\hline $\begin{array}{c}\mathrm{ITO} / \\
\mathrm{Nb}: \mathrm{SrTiO}_{3}\end{array}$ & Two & $\begin{array}{c}549- \\
630 \mathrm{~nm} \\
\end{array}$ & - & - & - & - & - & - & - & - & 5 \\
\hline $\mathrm{CH}_{3} \mathrm{NH}_{3} \mathrm{PbI}_{3}$ & Two & $V_{\mathrm{ds}}$ & $500 \mathrm{~nm}$ & - & - & - & - & - & - & - & 6 \\
\hline Gr/CNT & Three & $405 \mathrm{~nm}$ & $V_{\mathrm{g}}$ & - & - & - & - & - & - & - & 7 \\
\hline $\mathrm{WS}_{2}$ & Three & $532 \mathrm{~nm}$ & $V_{\mathrm{g}}$ & - & - & - & - & - & - & - & 8 \\
\hline WSe2 & Three & $473 \mathrm{~nm}$ & $V_{\mathrm{g}}$ & - & - & - & - & - & - & - & 9 \\
\hline $\mathrm{MoS}_{2} / \mathrm{PTCDA}$ & Three & $532 \mathrm{~nm}$ & $V_{\mathrm{g}}$ & - & - & - & - & - & - & - & 10 \\
\hline $\mathrm{MoS}_{2}$ & Three & $350 \mathrm{~nm}$ & $V_{\mathrm{g}}$ & - & - & - & - & - & - & - & 11 \\
\hline Si-NC/WSe2 & Three & 1342 nm & - & - & - & - & - & - & - & - & 12 \\
\hline Si NCs & Three & 1342 nm & $V_{\mathrm{g}}$ & - & - & $5 \%$ & - & $784 \times 100$ & $93 \%$ & 150 & 13 \\
\hline
\end{tabular}




\begin{tabular}{|c|c|c|c|c|c|c|c|c|c|c|c|}
\hline IGZO & Three & 365 nm & - & - & - & - & - & - & - & - & 14 \\
\hline IGZO & Three & 254 nm & - & - & - & - & - & - & - & - & 15 \\
\hline IGZO & Three & $405 \mathrm{~nm}$ & $V_{\mathrm{g}}$ & 4.24 & 8 & - & - & $785 \times 10$ & $62 \%$ & $>35000$ & 16 \\
\hline IGZO & Three & UV & $V_{\mathrm{g}}$ & - & - & - & - & CNN & $94.3 \%$ & $>400$ & 17 \\
\hline IGZO & Three & 405 nm & $V_{\mathrm{g}}$ & - & - & - & - & $784 \times 10$ & $82.6 \%$ & 300 & 18 \\
\hline $\begin{array}{c}\text { PQT-12+ } \\
\text { CsPbBr3 QDs }\end{array}$ & Three & $500 \mathrm{~nm}$ & - & - & - & - & - & - & - & - & 19 \\
\hline $\begin{array}{c}\text { Pentacene/ } \\
\text { PMMA/C } \mathrm{N}_{4}\end{array}$ & Three & 365 nm & - & - & - & - & - & - & - & - & 20 \\
\hline $\begin{array}{c}\text { Pentacene/ } \\
\text { PMMA/ } \\
\text { CsPbBr3 QDs }\end{array}$ & Three & $\begin{array}{c}365 \\
660 \mathrm{~nm}\end{array}$ & $V_{\mathrm{g}}$ & - & - & - & - & - & - & - & 21 \\
\hline $\mathrm{ZnO} \mathrm{NWs}$ & Three & 365 nm & - & - & - & - & - & - & - & - & 22 \\
\hline C8-BТВT & Three & $360 \mathrm{~nm}$ & - & - & - & - & - & - & - & - & 23 \\
\hline
\end{tabular}

-: not available; Pyr-GDY: pyrenyl graphdiyne; Gr: graphene; QDs: quantum dots; BP: black phosphorus; ITO: Indium-Tin oxide; CNT: carbon nanotubes; PTCDA: Perylene-3,4,9,10-tetracarboxylic dianhydride; Si-NC: Si-nanocrystal; IGZO: Indium gallium zinc oxide; PQT-12: poly(3,3didodecylquarterthiophene); PMMA: poly (methyl methacrylate); NWs: nanowires; Cc8-BTBT: 2,7-dioctyl[1]benzothieno[3,2b][1]benzothiophene. 


\section{Supplementary Note 1: Experimental Details}

\subsection{Synthesis of PbS quantum dots with excitonic peak at $980 \mathbf{n m}$}

A standard Schlenk technique was used in the reaction, and all reagents were purchased from Sigma Aldrich. $2 \mathrm{mmol}$ of PbO, $4.7 \mathrm{mmol}$ of oleic acid (OA) and $20 \mathrm{~mL}$ of 1- octadecene (ODE) were added into a three-necked flask and pumped at $95^{\circ} \mathrm{C}$ for $12 \mathrm{~h}$. And then, $15 \mathrm{ml}$ of ODE were added to the flask, followed by the raise of the reaction temperature to $120{ }^{\circ} \mathrm{C}$. After this step, $1 \mathrm{mmol}$ of hexamethyldisilathiane (HMS) mixed with $10 \mathrm{ml}$ of ODE were quickly injected. The heating stopped without removing the heating mantel to cool down the reaction slowly ( $60 \mathrm{~min}$ ). The quantum dots (QDs) were isolated after the addition of acetone and ethanol (5:1) and centrifugation, purified by two successive dispersions in toluene and precipitations with acetone/EtOH finally dispersed in anhydrous n-octane.

High-resolution transmission electron microscopy (HRTEM), X-ray diffraction (XRD) and Raman spectroscopy were used to characterize the prepared PbS QDs. Figure S1 features the morphology of the PbS QDs, with averge diameter of $\sim 4.1 \mathrm{~nm}$. The XRD peaks of the PbS QDs are well fitted with PDF (\#78-1054), indicating the successful preparation of PbS QDs (Figure S2).

The PbS QDs were deposited on the substrate via spin coating, followed by a ligand exchange process. Before spin coating, the $\mathrm{SiO}_{2} / \mathrm{Si}$ wafer or Polyethylene terephthalate (PET) film were first pre-treated by oxygen plasma cleaning to get a clean surface with good wettability, and the PbS QD solution was filtered to remove the aggregated particles. And then PbS QDs solution with concentration of $5 \mathrm{mg} / \mathrm{mL}$ in n-octane was spin coated on the substrates at $2500 \mathrm{rpm}$ for $15 \mathrm{~s}$ to form a uniform film (Figure S4). 2\% Vol. ethanedithiol (EDT) solution in acetonitrile was dropped to the PbS QD film and left for 30 s to exchange ligands, followed by the spin coating at $2500 \mathrm{rpm}$ for $15 \mathrm{~s}$. Finally, acetonitrile was dropped on the film and spin coated twice to wash off the excess EDT ligands. This process was repeated for 2 times to yield a PbS film with thickness of 12-16 nm (Figure S5d). The surface roughness of the film was $1.01 \mathrm{~nm}$. 


\subsection{Preparation of 1,3,6,8- tetraethynylpyrene monomers}

Synthetic route of 1,3,6,8-tetraethynylpyrene (TEP) monomers is illustrated in Figure S6a. Firstly, 1,3,6,8-tetrakis((trimethylsilyl)ethynyl)pyrene (TEP-TMS) was synthesized according to the reported method. ${ }^{24}$ In detail, $1.01 \mathrm{~g}$ pyrene was dissolved in $15 \mathrm{~mL}$ nitrobenzene, followed by adding of $3.52 \mathrm{~g}$ bromine to the solution at room temperature. The mixture was stirred at $120{ }^{\circ} \mathrm{C}$ for $4 \mathrm{~h}$ and the reaction mixture was filtered. The residue was washed with methanol and acetone and dried under reduced pressure to obtain pure 1,3,6,8tetrabromopyrene. Then, $250 \mathrm{mg}$ 1,3,6,8-tetrabromopyrene was suspended in $10 \mathrm{~mL}$ Et3 $\mathrm{N}$ and $3 \mathrm{~mL}$ toluene, and $68 \mathrm{mg} \mathrm{PdCl} 2\left(\mathrm{PPh}_{3}\right)_{2}$, $36 \mathrm{mg} \mathrm{CuI}$, and $50 \mathrm{mg} \mathrm{Ph}_{3} \mathrm{P}$ were added to the solution under an Ar atmosphere. $280 \mathrm{mg}$ (trimethylsilyl)ethyne was added to the mixture at $60{ }^{\circ} \mathrm{C}$, followed by stirring at $80{ }^{\circ} \mathrm{C}$ for $12 \mathrm{~h}$. The reaction mixture was diluted with $\mathrm{CH}_{2} \mathrm{Cl}_{2}$ and washed with water. The organic layer was dried by $\mathrm{MgSO}_{4}$, and the solvents were removed under reduced pressure. After a column chromatography on silica gel (hexane) and reprecipitation from $\mathrm{CH}_{2} \mathrm{Cl}_{2} / \mathrm{MeOH}$, pure form 1,3,6,8-TEP-TMS was obtained successfully.

Then, $2.94 \mathrm{mg}$ 1,3,6,8-TEP-TMS was dissolved into $50 \mathrm{~mL}$ tetrahydrofuran, and $50 \mu \mathrm{L}$ tetrabutylammonium fluoride was added to the solution under an Ar atmosphere. After stirring at $0{ }^{\circ} \mathrm{C}$ for 20 min under an Ar atmosphere, the reaction mixture was diluted with ethyl acetate and washed with saturated salt water for three times. The organic layer was dried by $\mathrm{MgSO}_{4}$ and the solvents were removed under reduced pressure. The 1,3,6,8-TEP monomers were obtained and should be used immediately.

Figure S6b illustrates the structure of Pyr-GDY, and its Raman and XPS spectra are shown in Figure S7 and S8, respectively.

\subsection{Device fabrication}

PbS QD film with thickness of $~ 14 \mathrm{~nm}$ was first deposited on $\mathrm{SiO}_{2} / \mathrm{Si}$ or flexible PET film, followed by the transfer of chemical vapor deposition (CVD)-grown monolayer graphene on top of PbS film using a poly (methyl methacrylate) (PMMA)-assisted "electrochemical bubbling” method. ${ }^{25}$ The transferred graphene film was patterned into a rectangular channel by laser-direct lithography (miDALIX) and oxygen plasma etching. And then, the source and drain electrodes $(\mathrm{Cr} / \mathrm{Au} 10 \mathrm{~nm} / 50 \mathrm{~nm})$ of the device were fabricated on graphene channel by laser- 
direct lithography and thermal evaporation. Finally, a uniform Pyr-GDY film was synthesized on top of graphene channel via a van der Waals epitaxial strategy. ${ }^{26}$ In this work, the effective length and width of the device were 10 and $50 \mu \mathrm{m}$, respectively.
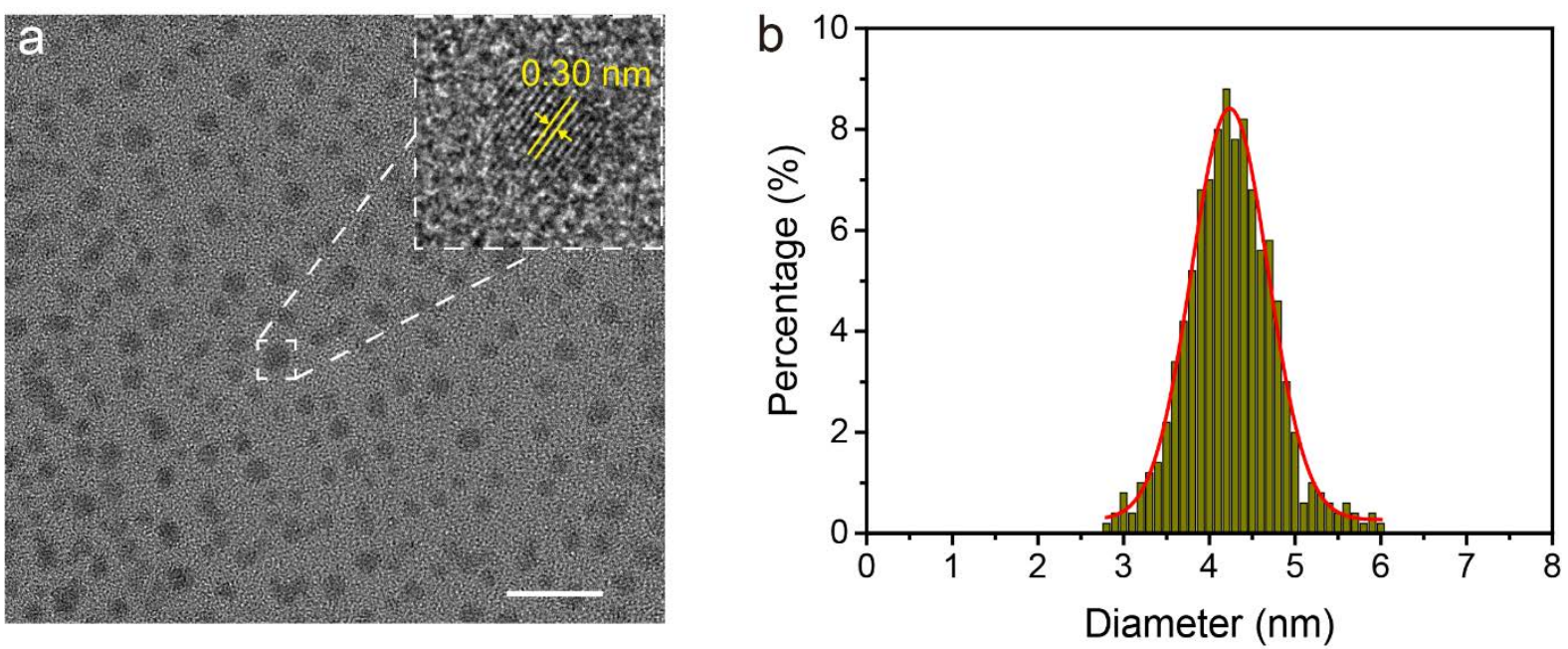

Figure S1. Morphology characterization of PbS QDs. (a) HREEM image of the prepared PbS QDs. Scale bar, $20 \mathrm{~nm}$. (b) Statistics of the size of the prepared PbS QDs.

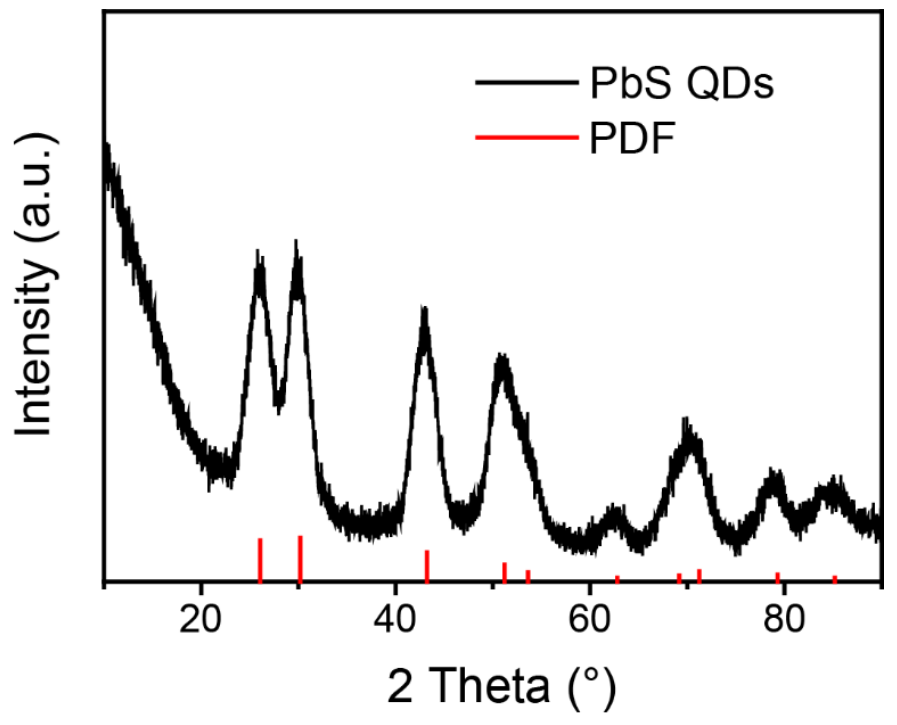

Figure S2. XRD pattern of PbS QDs. 


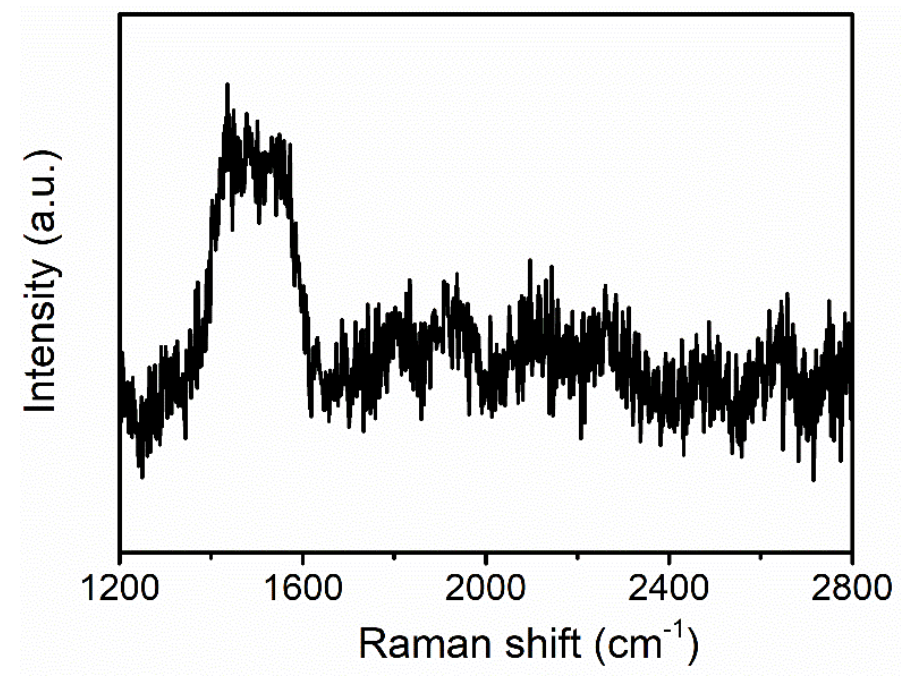

Figure S3. Raman spectrum of PbS QDs.
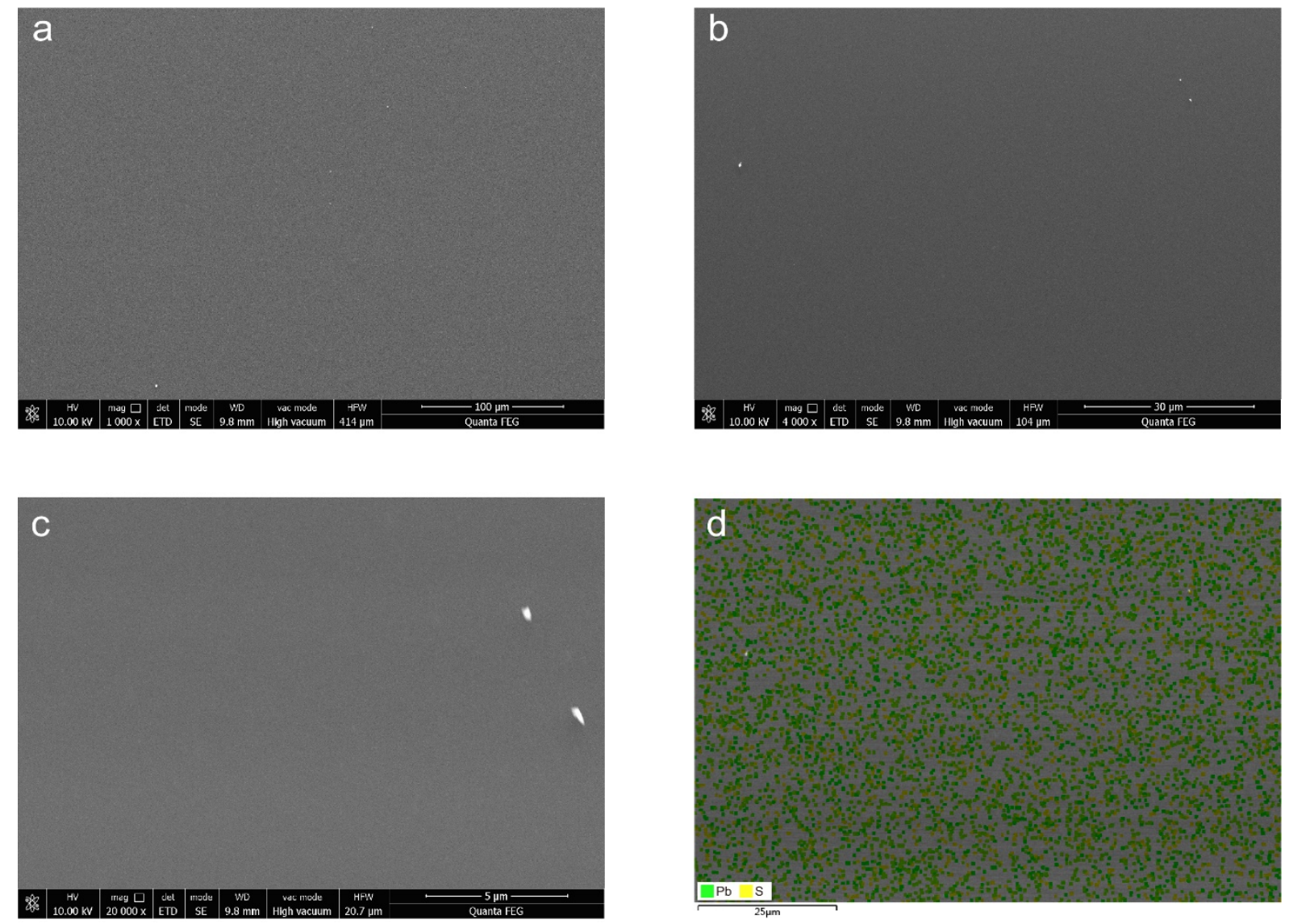

Figure S4. SEM image of PbS QD film deposited on $\mathrm{SiO}_{2} / \mathrm{Si}$ wafer via spin coating with magnification of (a) $1000 \times$, (b) $4000 \times$ and (c) $20000 \times$, respectively. The uniform contrast in these films demonstrate the high uniformity of the deposited PbS QD film. (d) EDS image of the PbS QD film corresponding to the area as shown in (b), demonstrating uniform distributed $\mathrm{Pb}$ and $\mathrm{S}$ elements. 

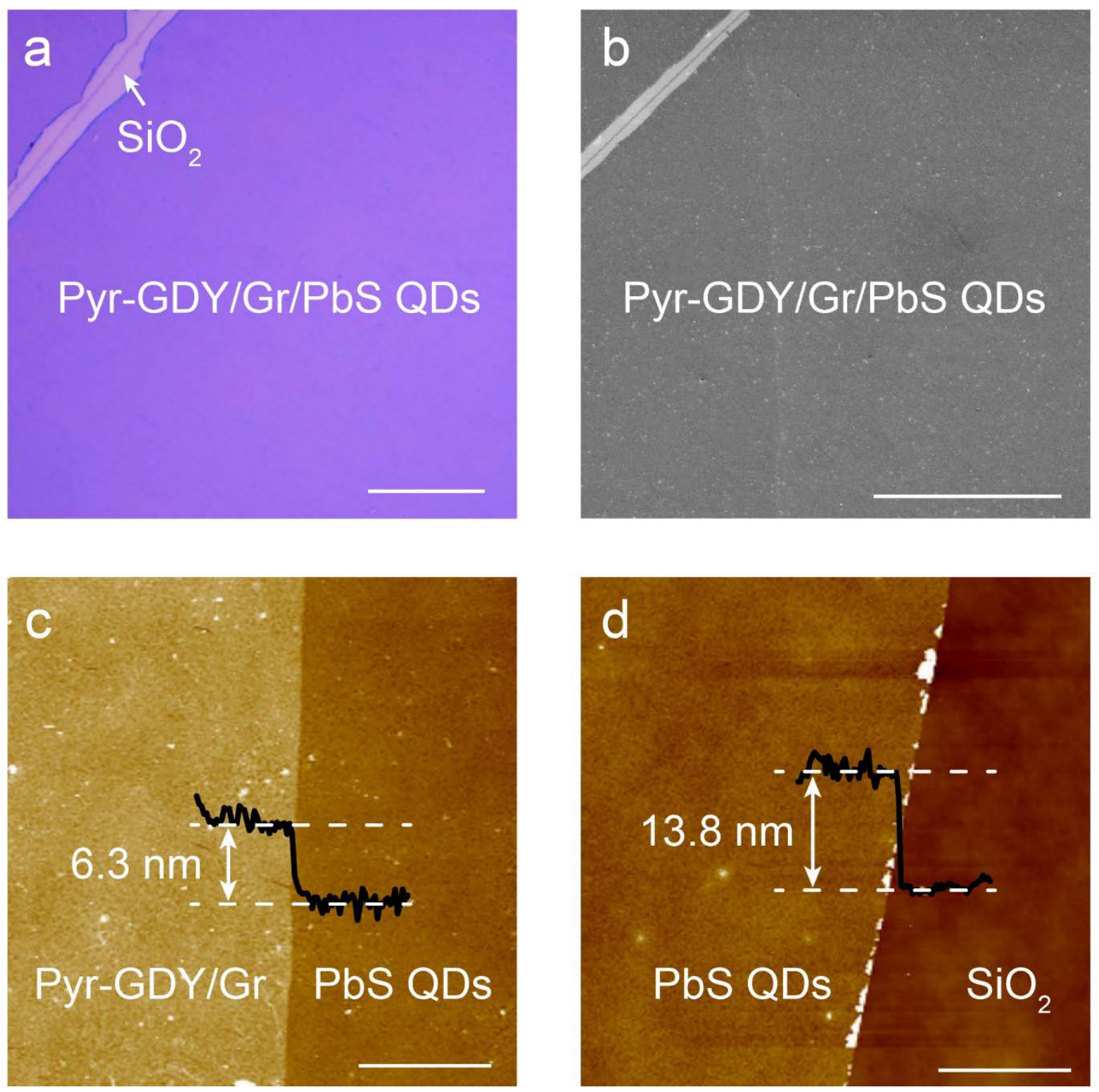

Figure S5. Surface morphology of Pyr-GDY/Gr/PbS-QDs heterostructure on $\mathrm{SiO}_{2} / \mathrm{Si}$. (a) $\mathrm{OM}$ and (b) SEM images of Pyr-GDY/Gr/PbS-QDs heterostructure. Scale bars, $100 \mu \mathrm{m}$. (c,d) AFM images of (c) Pyr-GDY/Gr on PbS film and (d) $\mathrm{PbS}$ film on $\mathrm{SiO}_{2}$ substrate, respectively. Scale bars, $5 \mu \mathrm{m}$. 

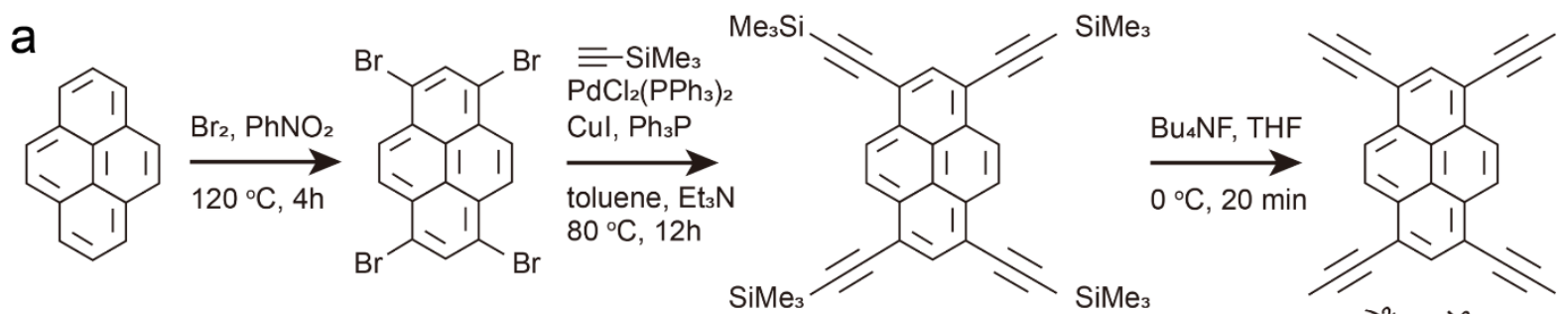

$\mathrm{SiMe}_{3}$
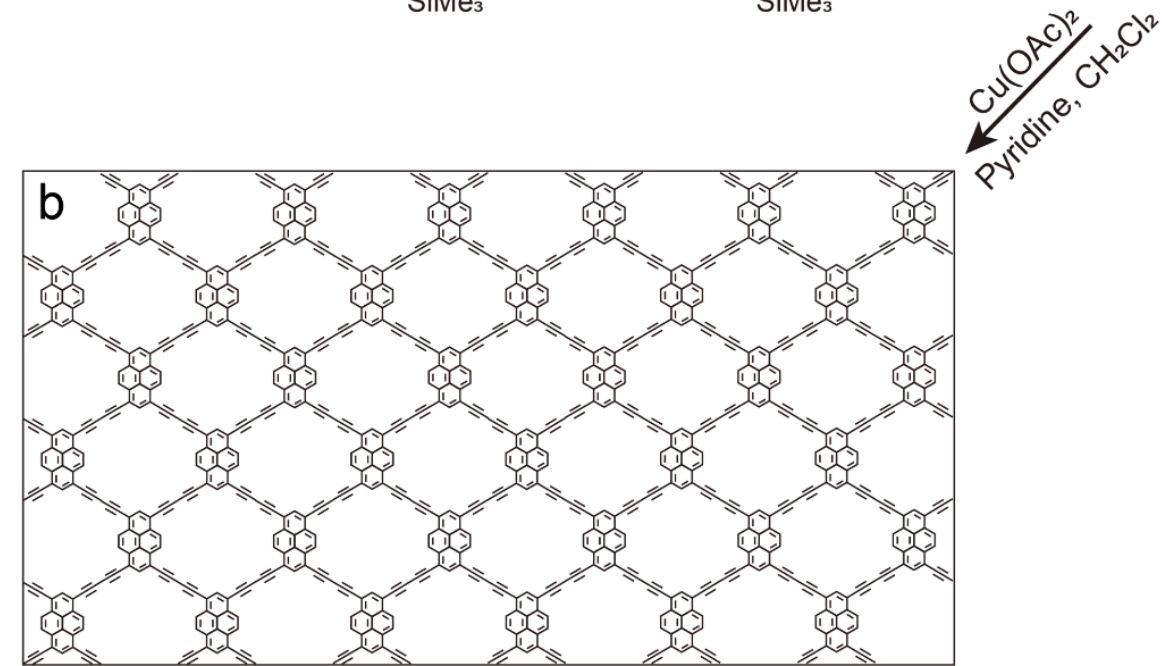

Figure S6. Synthesis process of Pyr-GDY. (a) Synthetic route for 1,3,6,8-TEP monomers. (b) Schematic illustration of Pyr-GDY.

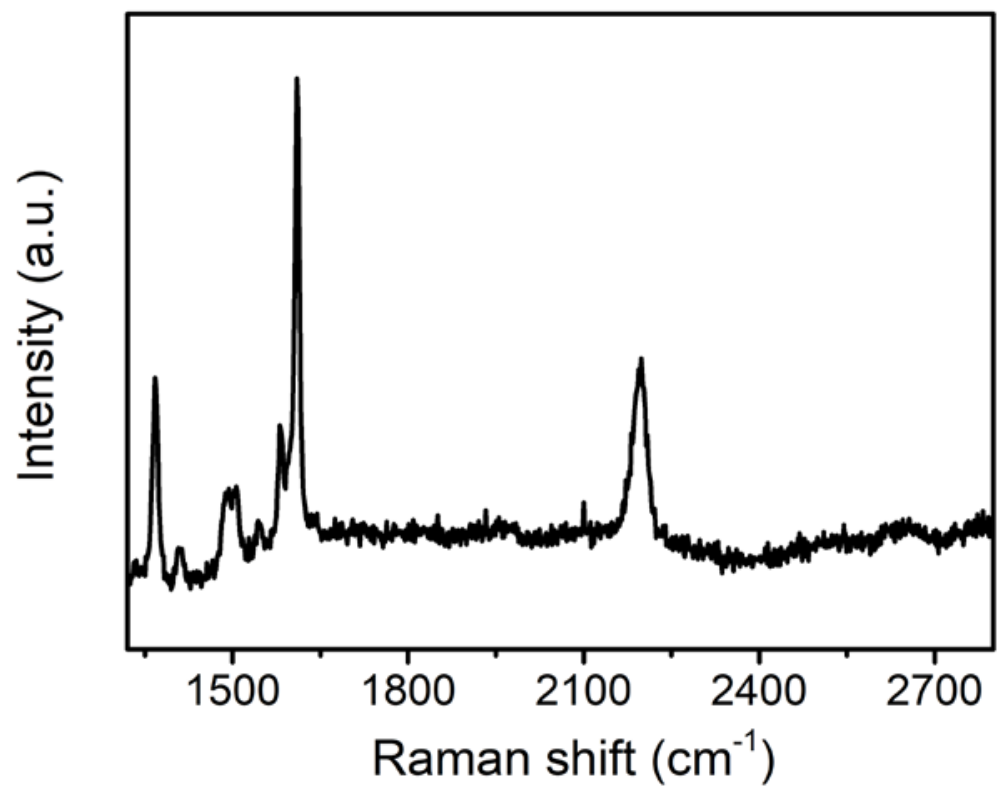

Figure S7. Raman spectrum of Pyr-GDY. 

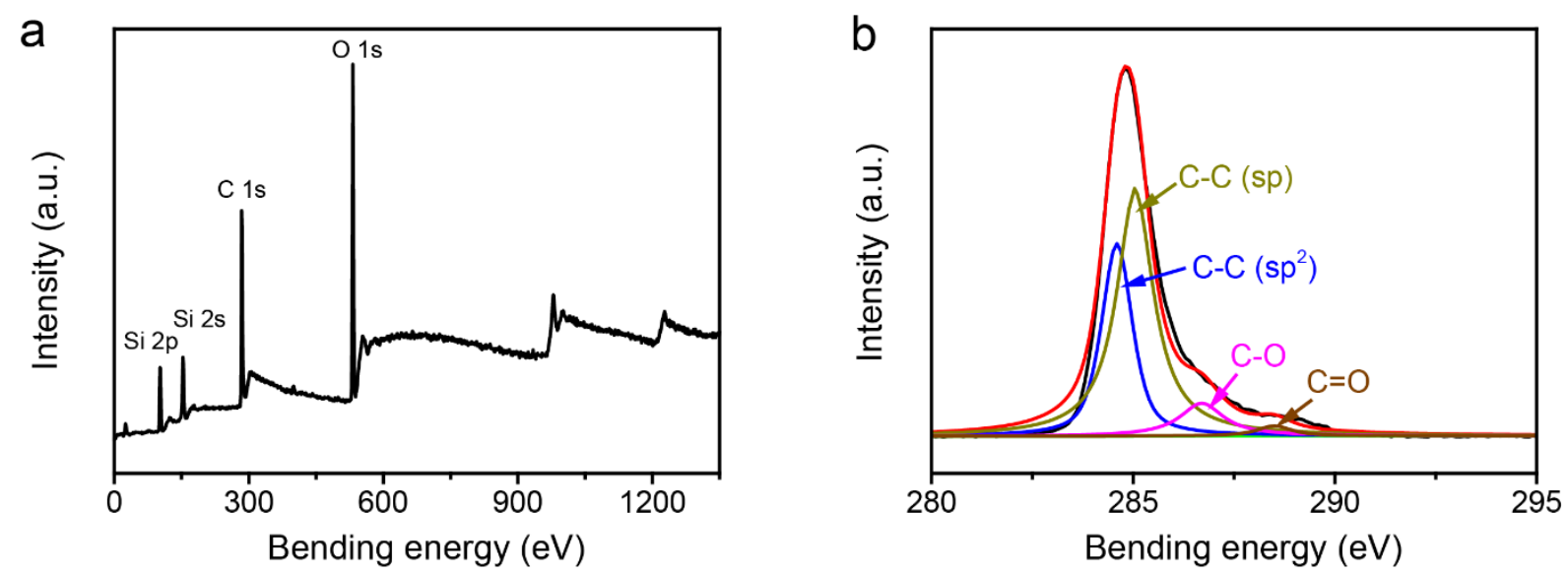

Figure S8. (a) XPS spectra of Pyr-GDY and (b) its corresponding C 1s peak.

a

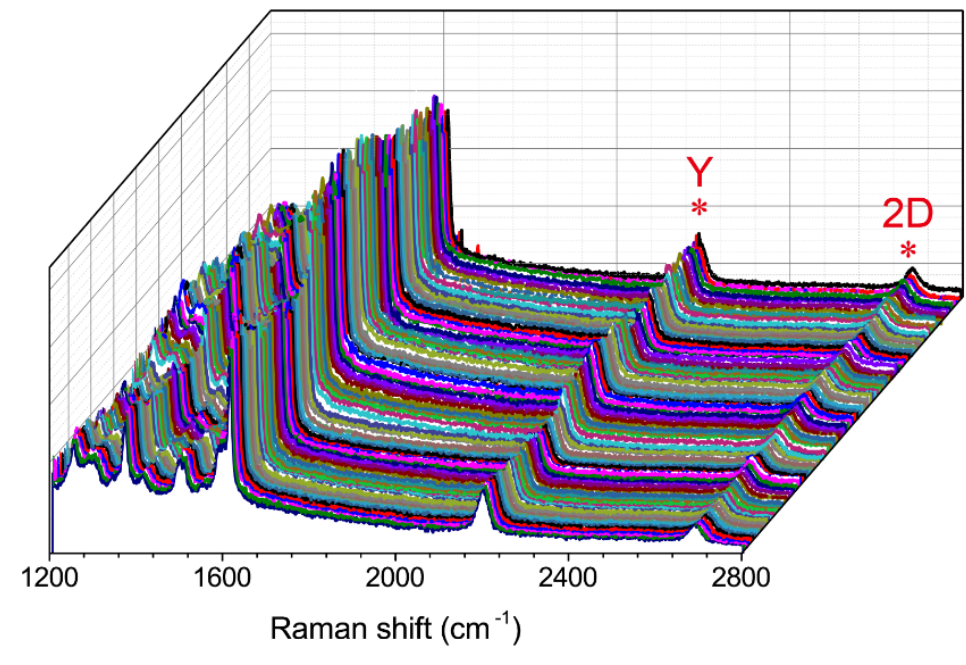

b

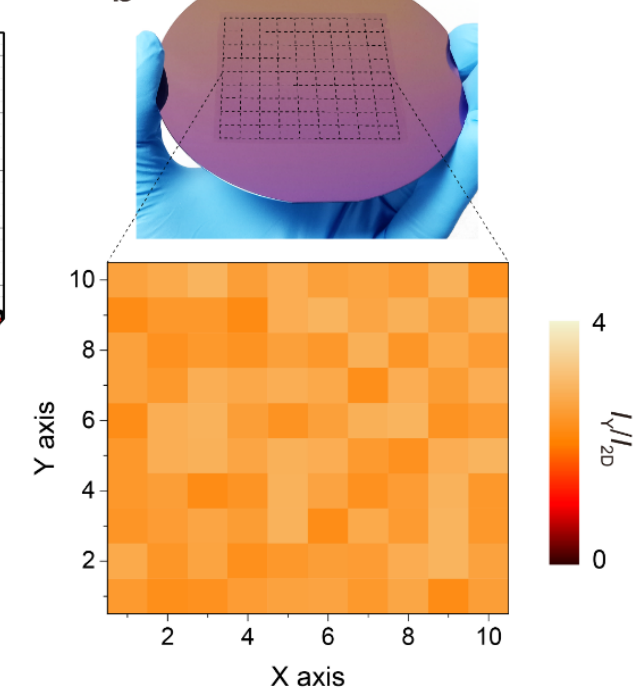

Figure S9. Raman spectra characterization of the fabricated wafer-scale Pyr-GDY/Gr/PbSQDs heterostructure. (a) Raman spectra measured at different points of the wafer-scale PyrGDY/Gr/PbS film. (b) Raman mapping for the intensity ratio of the Y band $\left(2194 \mathrm{~cm}^{-1}\right)$ and 2D band $\left(2678 \mathrm{~cm}^{-1}\right)$, i.e. $I_{\mathrm{Y}} / I_{2} \mathrm{D}$, extracted from the Raman spectra measured at the corresponding points marked in the upper photograph. The homologous Raman spectra and uniform Raman mapping indicate the high uniformity of the synthesized Pyr-GDY/Gr/PbSQDs heterostructure in macroscopic scale. 

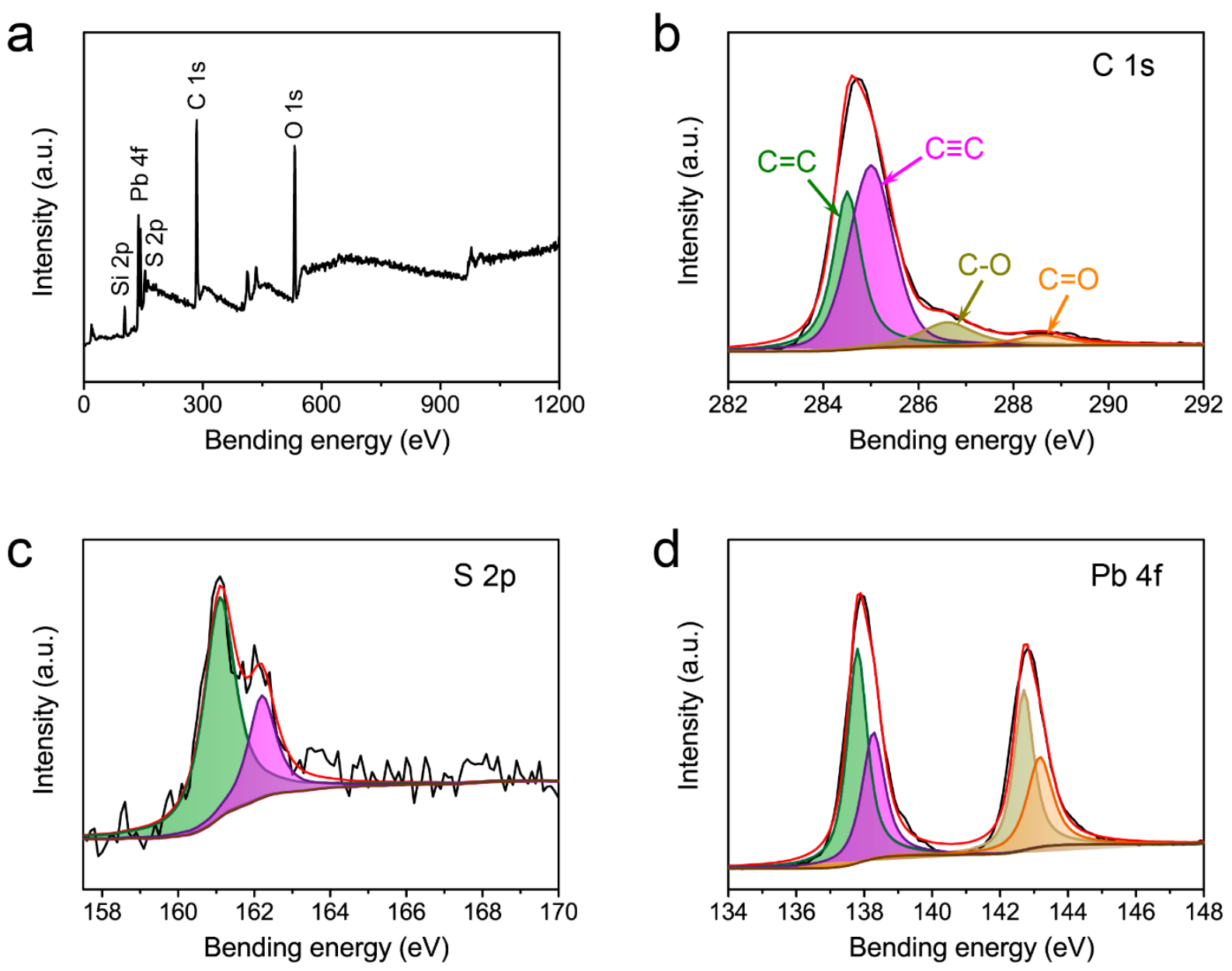

Figure S10. XPS spectra for the Pyr-GDY/Gr/PbS-QDs heterostructure. (a) XPS spectrum for the heterostructure on $\mathrm{SiO}_{2} / \mathrm{Si}$ substrate. (b-d) C 1s, S 2p and Pb 4f peaks of the XPS spectra for the Pyr-GDY/Gr/PbS-QDs. 

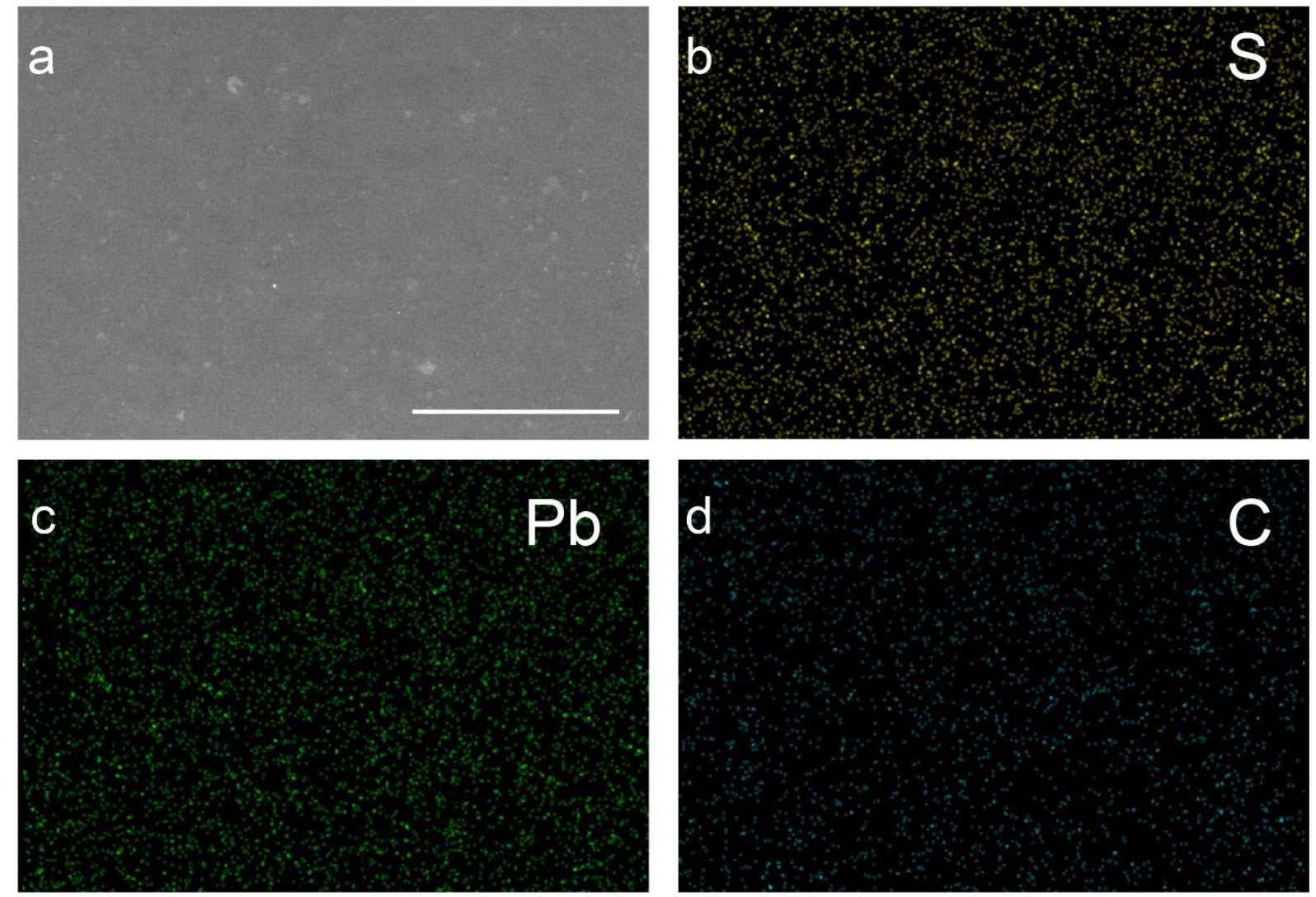

Figure S11. EDS for the Pyr-GDY/Gr/PbS-QDs heterostructure. (a) SEM image and corresponding element mapping images of (b) S, (c) $\mathrm{Pb}$ and (d) $\mathrm{C}$ in the heterostructure. Scale bar, $25 \mu \mathrm{m}$.
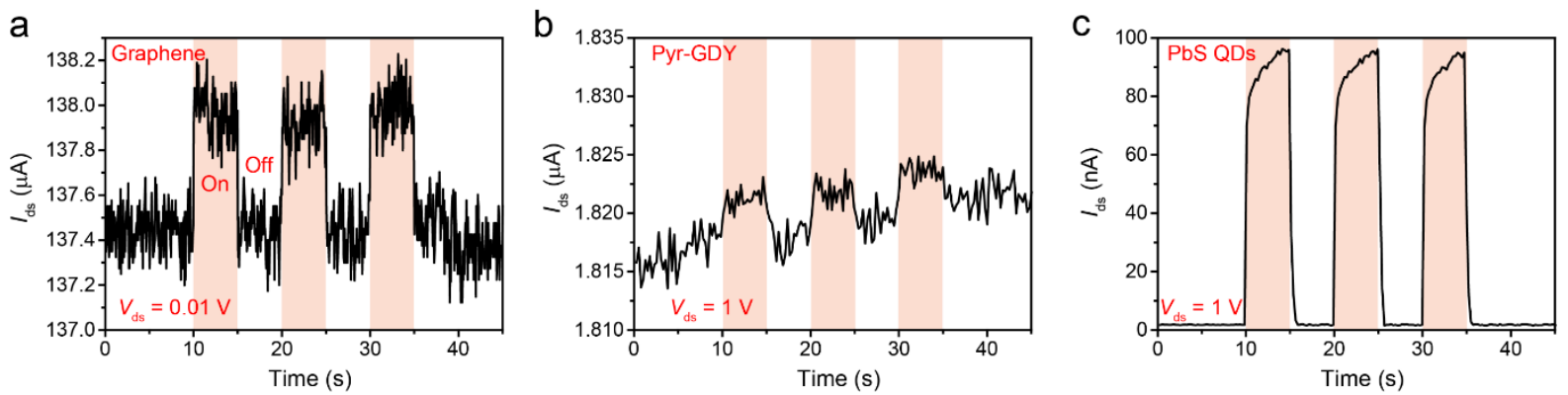

Figure S12. Photoresponses of (a) graphene-based, (b) Pyr-GDY-based and (c) PbS QDsbased devices illuminated by a $980 \mathrm{~nm}$ laser with an intensity of $800 \mathrm{~mW} \mathrm{~cm}{ }^{-2}$. The drain voltage for graphene-based device was $0.01 \mathrm{~V}$, while it was $1 \mathrm{~V}$ for Pyr-GDY-based and PbS QDs-based devices. The photoresponse of graphene-based device was only $\sim 0.5 \mu \mathrm{A}$, owing to the small responsivity of pristine graphene (in range of $\mathrm{mA} \mathrm{W}^{-1}$ ) and weak effective light intensity $(4 \mu \mathrm{W})$. For Pyr-GDY-based device, since the absorption of Pyr-GDY at $980 \mathrm{~nm}$ is almost zero, the photoresponse is very weak. A significant photoresponse is observed for $\mathrm{PbS}$ QDs-based device, owing to its strong absorption at $980 \mathrm{~nm}$. 


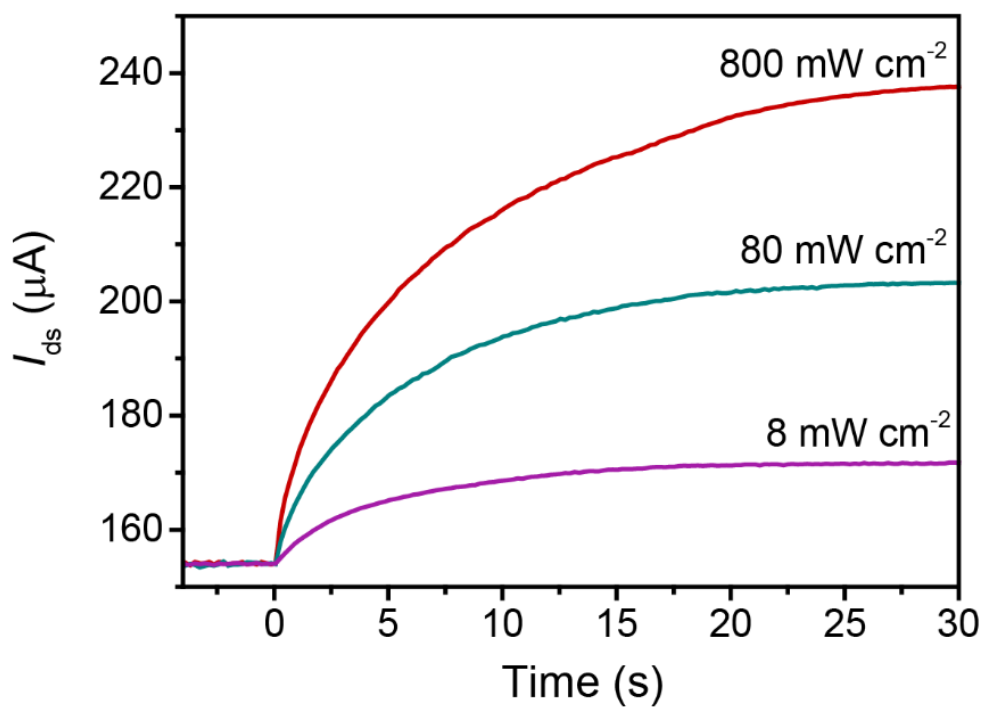

Figure S13. The dynamic responses of the Pyr-GDY/Gr/PbS-QD-based device under light illumination of $980 \mathrm{~nm}$ with intensities of 800,80 and $8 \mathrm{~mW} \mathrm{~cm}^{-2}$, respectively, demonstrating light-intensity- and time-dependent characteristics. 


\section{Supplementary Note 2: Energy Band Alignment}

The Pyr-GDY/Gr/PbS-QD heterostructure has two interfaces - Pyr-GDY/Gr interface and $\mathrm{Gr} / \mathrm{PbS}$ QDs interface. The mismatch of the work function for these three layers induces the band bending at the Pyr-GDY/Gr and Gr/PbS QDs interfaces. Here we used two different pathways to analyze the energy band alignment of the Pyr-GDY/Gr/PbS-QD heterostructure. In the Pathway 1, we first synthesized Pyr-GDY/Gr heterostructure, and then transferred the heterostructure onto the PbS QD film to construct Pyr-GDY/Gr/PbS-QD heterostructure. In the Pathway 2, graphene was first transferred onto PbS QD film, forming a Gr/PbS interface, and then Pyr-GDY was synthesized on the surface of graphene to construct Pyr-GDY/Gr/PbS-QD heterostructure. The Fermi levels of the samples were measured by Kelvin probe force microscopy (KPFM) using Au as calibration (5.1 eV), and the results are shown in Figure S14. Other characterizations, such as the transfer curves and Raman spectra, were used to demonstrate the change of the doping level of graphene in pathway 1 and 2 (Figure S16 and S17).

\subsection{Pathway 1}

In this pathway, Pyr-GDY/Gr heterostructure was constructed first. Figure S15a illustrates the band energy of PbS QDs, graphene and Pyr-GDY before contact. The Fermi levels $\left(E_{\mathrm{F}}\right)$ of Pyr-GDY and pristine graphene are $5.05 \mathrm{eV}$ (Figure S14a) and $4.6 \mathrm{eV},{ }^{27}$ respectively. And the bandgap of Pyr-GDY measured via a Tauc method is $2.08 \mathrm{eV}$ (Figure S18). Owing to the mismatch of the work functions between Pyr-GDY and graphene, holes in Pyr-GDY would transfer to graphene, leading to a downward band bending at the Pyr-GDY/Gr interface. After the contact, the aligned Fermi level of the Pyr-GDY/Gr heterostructure was measured to be $5.01 \mathrm{eV}$ (Figure S14b), indicating a heavy p-doping of graphene channel.

As shown in Figure S14d, the Fermi level of PbS QDs is $4.81 \mathrm{eV}$, which is lower than that of Pyr-GDY/Gr heterostructure (Figure S15b). Therefore, electrons would transfer from PbS QDs to graphene, resulting in an upward bending at the PbS/Gr interface (Figure S15d). It is worth noting that the injection of electrons from PbS QDs to graphene leads to the rise of graphene Fermi level, breaking the balance between graphene and Pyr-GDY. Thus with the 
contact of PbS QDs to Pyr-GDY/Gr heterostructure, more holes would transfer from Pyr-GDY to graphene, inducing a larger downward bending at the Pyr-GDY/Gr interface. Finally, the Fermi levels of Pyr-GDY, graphene and PbS QDs would align again and reach a new equilibrium state, and the aligned Fermi level was measured to be $4.91 \mathrm{eV}$ (Figure S14c).

The change of the Fermi level of graphene in Pathway 1 can also be demonstrated by the transfer curves and Raman spectra. As shown in Figure S16a and S17a, the Dirac voltage of graphene was larger than $90 \mathrm{~V}$, and the Raman G $\left(1593 \mathrm{~cm}^{-1}\right)$ and $2 \mathrm{D}\left(2684 \mathrm{~cm}^{-1}\right)$ bands of graphene exhibited a significant redshift in comparison with that of pristine graphene $\left(\omega_{\mathrm{G}}=\right.$ $\left.1582 \mathrm{~cm}^{-1}, \omega_{2 \mathrm{D}}=2670 \mathrm{~cm}^{-1}\right)$, indicating that graphene was heavily $p$-doped by the Pyr-GDY.28 After the contact of PbS QDs, the Dirac voltage shifted back to $40 \mathrm{~V}$, and the Raman G and 2D bands exhibited a relative blueshift $\left(1589 \mathrm{~cm}^{-1}\right.$ and $\left.2678 \mathrm{~cm}^{-1}\right)$ in comparison with that before PbS QDs contact, which indicates that the p-doping level of graphene was reduced by the injection of electrons from PbS QDs.

\subsection{Pathway 2}

In this pathway, graphene was first transferred onto $\mathrm{PbS}$ film, forming a $\mathrm{Gr} / \mathrm{PbS}$ interface. Since the Fermi levels of pristine graphene and PbS QDs are $4.6 \mathrm{eV}$ and $4.81 \mathrm{eV}$, respectively, holes would transfer from PbS QDs to graphene after contact, leading to a downward band bending at the Gr/PbS interface (Figure S15c). The aligned Fermi level of Gr/PbS QDs heterostructure was measured to be $4.74 \mathrm{eV}$ (Figure S14e). It is worth noting that a downward bending is obtained at this stage, which is opposite to the upward bending discussed above. This is just the intermediate state before contacting Pyr-GDY.

When Pyr-GDY contacts Gr/PbS QDs heterostructure, the mismatch between the Fermi levels of Pyr-GDY (5.05 eV) and Gr/PbS QDs (4.74 eV) induced the transfer of holes from Pyr-GDY to graphene, forming a downward bending at the Pyr-GDY/Gr interface. With the injection of a large number of holes to graphene, the Fermi level of graphene dropped significantly, exceeding $4.81 \mathrm{eV}$ (Fermi level of PbS QDs). As a result, electrons would transfer from PbS QDs to graphene to reach a new equilibrium state, and finally the aligned Fermi level of Pyr-GDY/Gr/PbS-QD heterostructure is $4.93 \mathrm{eV}$ (Figure S14f), lower than the Fermi level of pristine PbS QDs. That is, more electrons are injected into graphene after the contact of Pyr- 
GDY, in comparison with the injected holes before the contact of Pyr-GDY. Thus the downward bending gradually transforms to upward bending (Figure S15d), which is consistent with that in Pathway 1 discussed above.

The change of the Fermi level of graphene in Pathway 2 can also be demonstrated by the transfer curves and Raman spectra. The Dirac voltage of Gr/PbS QDs was measured to be 22 V (Figure S16b), and the Raman G and 2D bands were red-shifted to $1587 \mathrm{~cm}^{-1}$ and $2676 \mathrm{~cm}^{-}$ 1, respectively (Figure S17b), indicating that graphene was $p$-doped by PbS QDs. After the contact of Pyr-GDY, the Dirac voltage further shifted to $40 \mathrm{~V}$, and the Raman G and 2D bands exhibited a further red-shift (1589 $\mathrm{cm}^{-1}$ and $2678 \mathrm{~cm}^{-1}$ ), which indicates that graphene was further $p$-doped by Pyr-GDY.

Actually, regardless the intermediate states, we can analysis the energy band alignment just on the basis of the Fermi levels of Pyr-GDY, graphene and PbS QDs before and after contact (Figure S15a,d). Before contact, the Fermi levels of these three layers are $5.05 \mathrm{eV}, 4.6 \mathrm{eV}$ and $4.81 \mathrm{eV}$, respectively, whereas they are aligned to $\sim 4.91 \mathrm{eV}$ after contact. That is, Pyr-GDY transfers holes and PbS QDs transfer electrons to graphene channel, inducing a downward band bending at Pyr-GDY/Gr interface and an upward bending at Gr/PbS interface, respectively. 

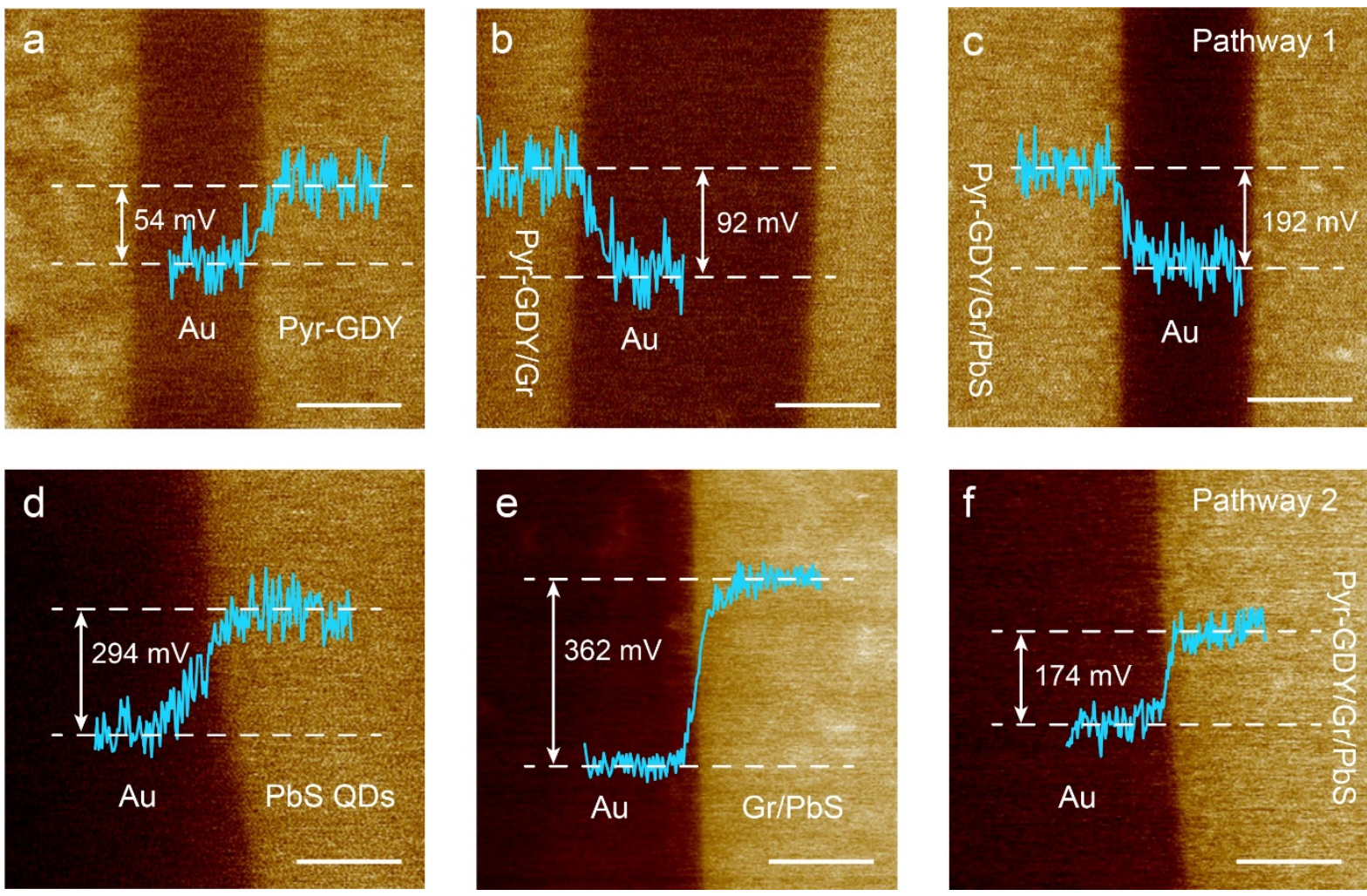

Figure S14. KPFM images for (a) Pyr-GDY, (b) Pyr-GDY/Gr, (c) Pyr-GDY/Gr/PbS heterostructure fabricated via Pathway 1, and (d) PbS QDs, (e) Gr/PbS, (f) Pyr-GDY/Gr/PbS heterostructure fabricated via Pathway 2, respectively. Scale bars, $5 \mu \mathrm{m}$. 


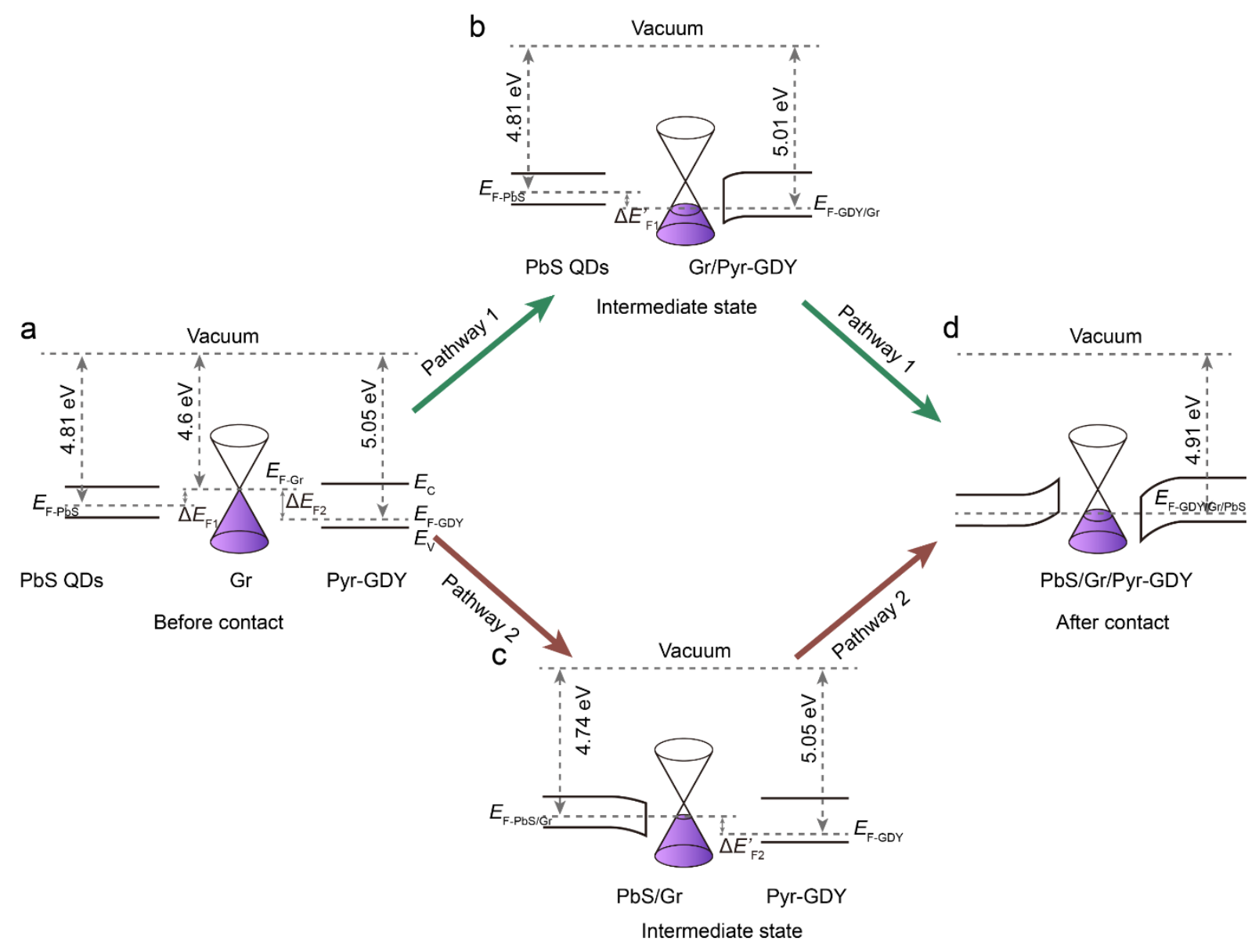

Figure S15. Energy band alignment for (a) individual PbS QDs, graphene and Pyr-GDY before contact, (b) graphene and Pyr-GDY contact first, (c) PbS QDs and graphene contact first, and (d) $\mathrm{PbS} / \mathrm{Gr} / \mathrm{Pyr}-\mathrm{GDY}$ heterostructure after contact, respectively. After contact, downward band bending is formed at the Gr/Pyr-GDY interface, while an upward bending is formed at the $\mathrm{PbS} / \mathrm{Gr}$ interface.
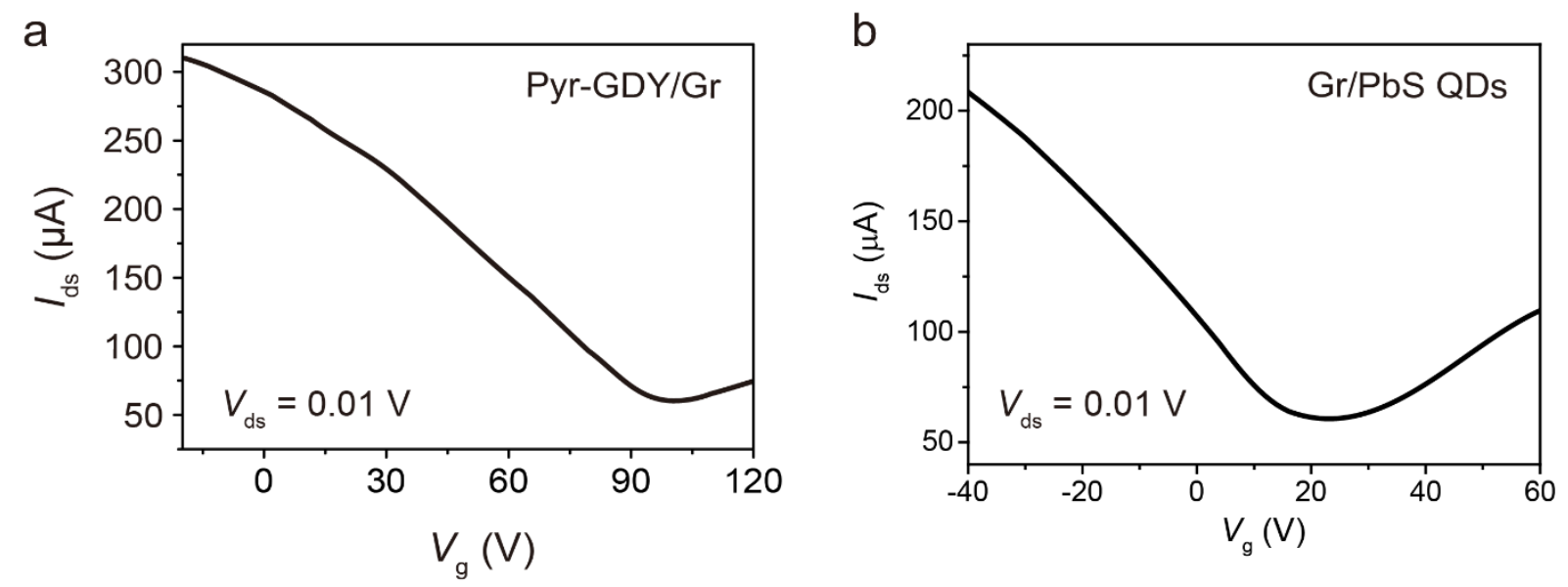

Figure S16. The transfer curves of the FET devices based on (a) Pyr-GDY/Gr and (b) Gr/PbS QDs, respectively. The Dirac voltages in (a) and (b) are $90 \mathrm{~V}$ and $22 \mathrm{~V}$, respectively. 

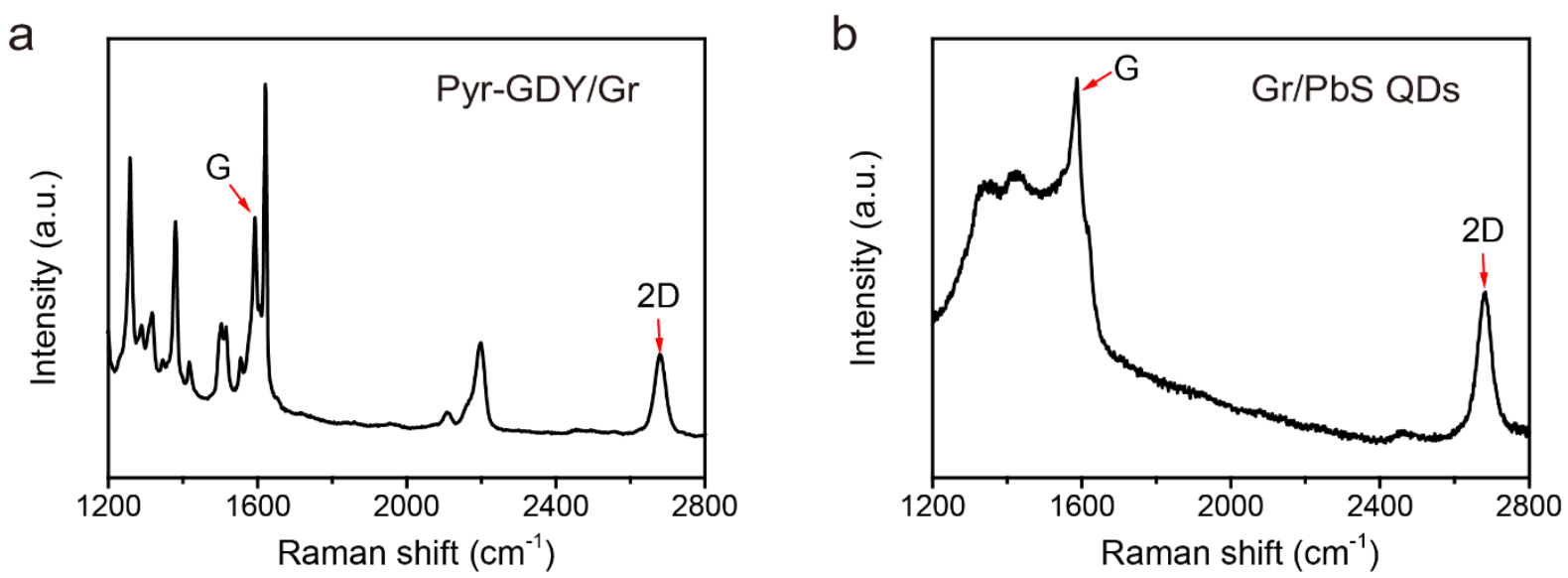

Figure S17. Raman spectra of (a) Pyr-GDY/Gr and (b) Gr/PbS QDs, respectively. The marked peaks at (a) $1593 \mathrm{~cm}^{-1}$ and $2684 \mathrm{~cm}^{-1}$ and (b) $1587 \mathrm{~cm}^{-1}$ and $2676 \mathrm{~cm}^{-1}$ correspond to the $\mathrm{G}$ and $2 \mathrm{D}$ bands of graphene, exhibiting a redshift in comparison with those of intrinsic graphene.

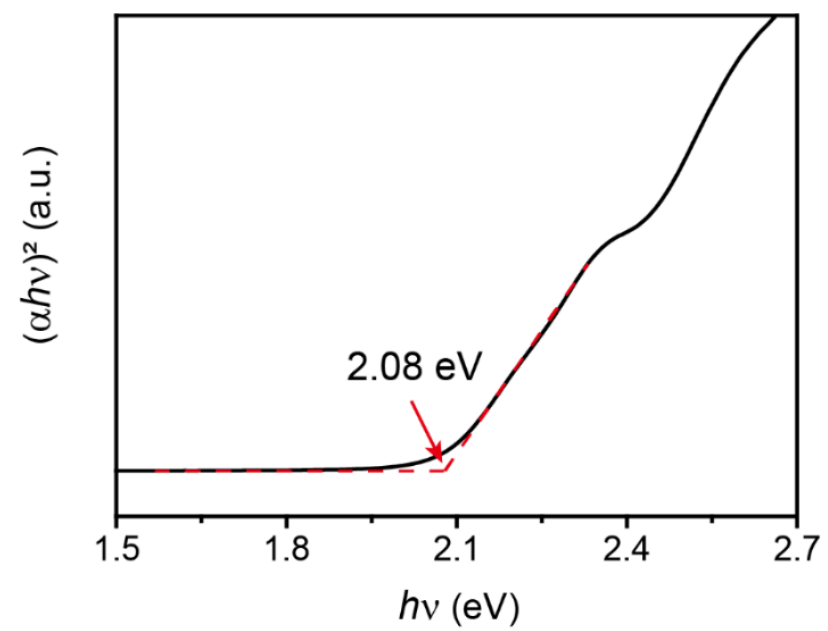

Figure S18. Tauc plot for the Pyr-GDY film, indicating a bandgap of $2.08 \mathrm{eV}$. 

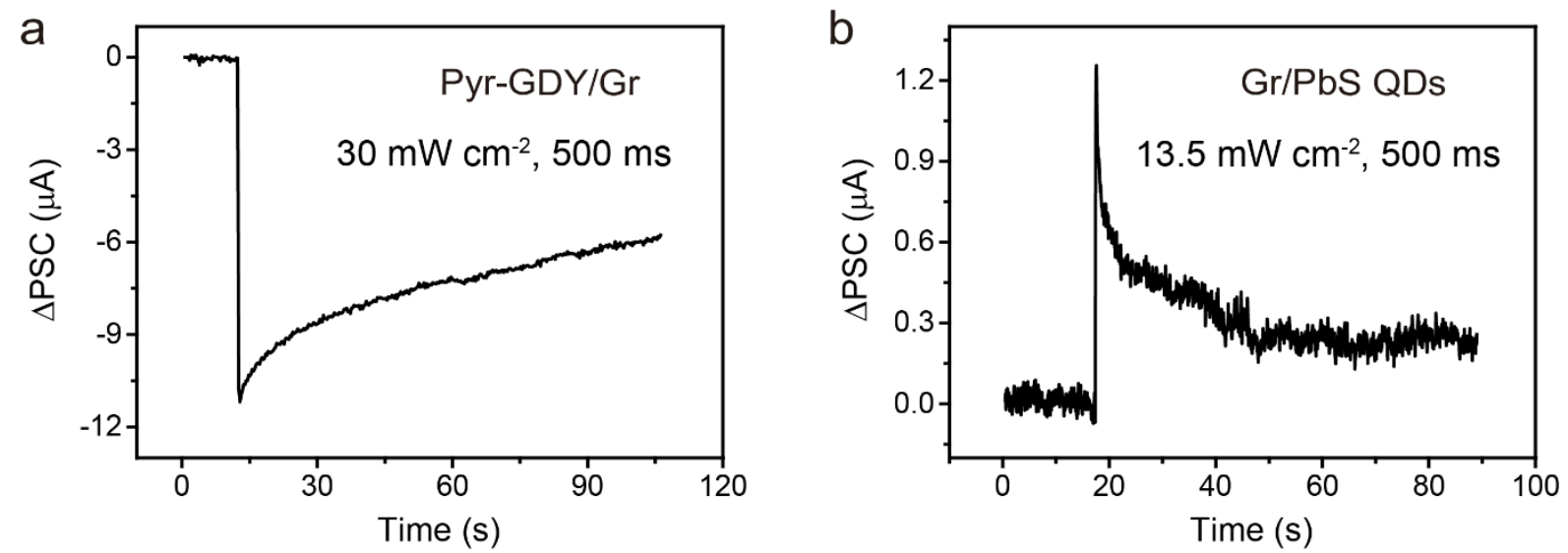

Figure S19. Dynamic responses of the devices based on (a) Pyr-GDY and (b) Gr/PbS QDs triggered by a $450 \mathrm{~nm}$ optical pulse. To emulate the actual illumination intensity for Pry-GDY and PbS QDs in Pyr-GDY/Gr/PbS-QD-based device, the light intensities for (a) Pyr-GDY/Grbased device and (b) Gr/PbS QDs-based device were set as $30 \mathrm{~mW} \mathrm{~cm}^{-2}$ and $13.5 \mathrm{~mW} \mathrm{~cm}^{-2}$ (30 $\mathrm{mW} \mathrm{cm}^{-2} \times 45 \%$ ), respectively. The IPSC response of the Pyr-GDY/Gr-based device is much stronger than the EPSC response of the Gr/PbS QDs-based device. $V_{\mathrm{ds}}=0.01 \mathrm{~V}$.
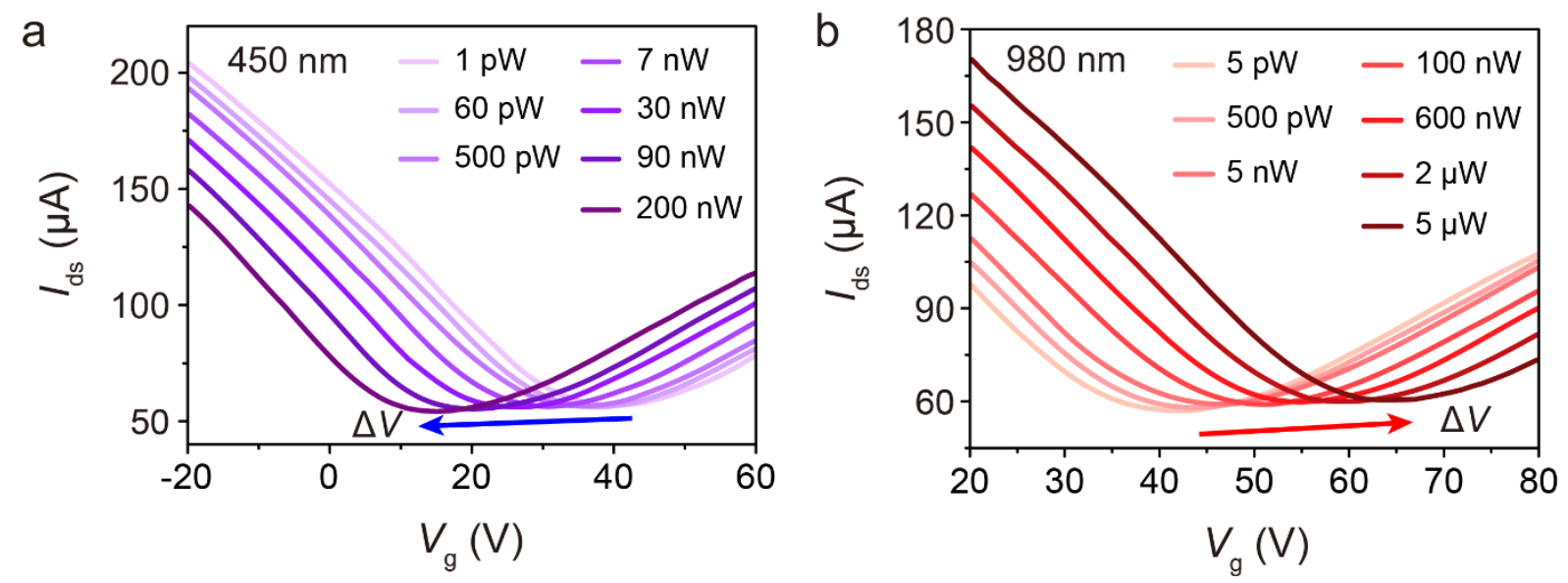

Figure S20. Transfer characteristics of the device based on Pyr-GDY/Gr/PbS-QDs under light illumination of (a) $450 \mathrm{~nm}$ and (b) $980 \mathrm{~nm}$ with different intensities. The Dirac voltage shifts to lower gate voltage with the increase of light intensity for $450 \mathrm{~nm}$ irradiation, and a positive shift of Dirac voltage is observed for increasing $980 \mathrm{~nm}$ irradiation intensity. $V_{\mathrm{ds}}=0.01 \mathrm{~V}$. 

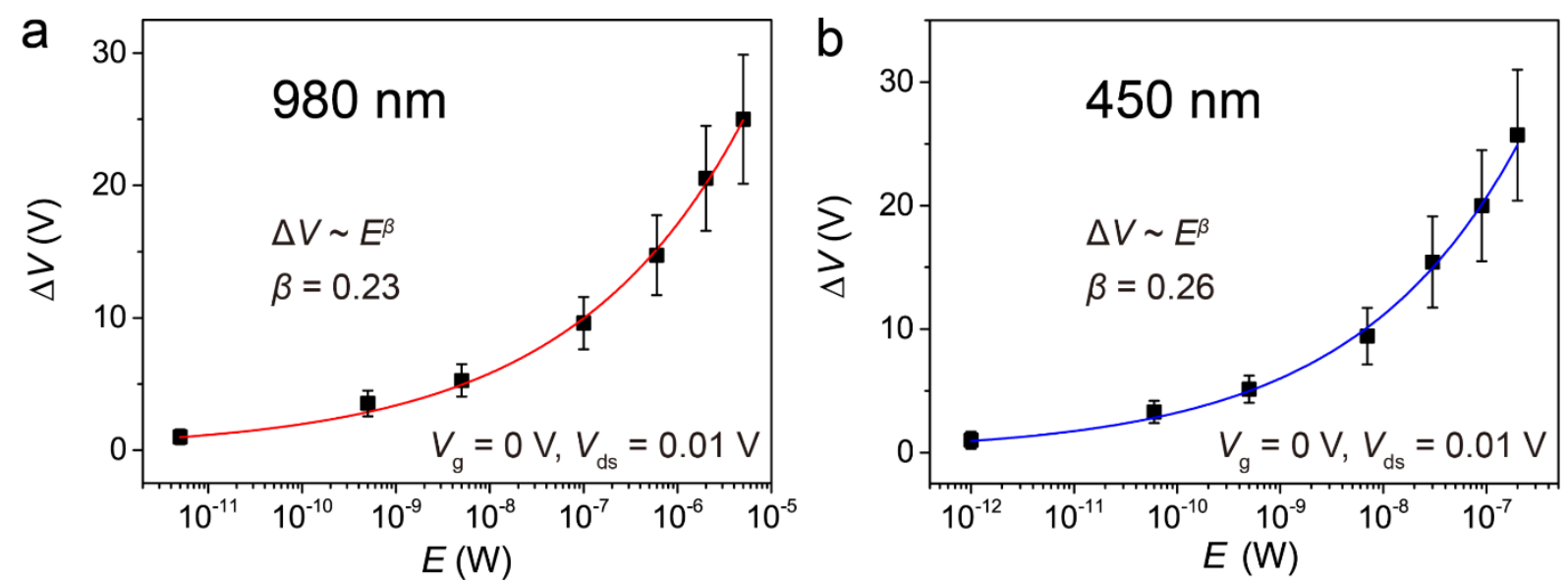

Figure S21. Horizontal shift of the Dirac voltage as a function of effective illumination intensity for (a) $980 \mathrm{~nm}$ and (b) $450 \mathrm{~nm}$ irradiation. The curves can be fitted by $\Delta V \sim P^{\beta}$ with $\beta=0.26$ and 0.23 for $450 \mathrm{~nm}$ and $980 \mathrm{~nm}$ light irradiation, respectively. $V_{\mathrm{g}}=0 \mathrm{~V}$ and $V_{\mathrm{ds}}=$ $0.01 \mathrm{~V}$. 

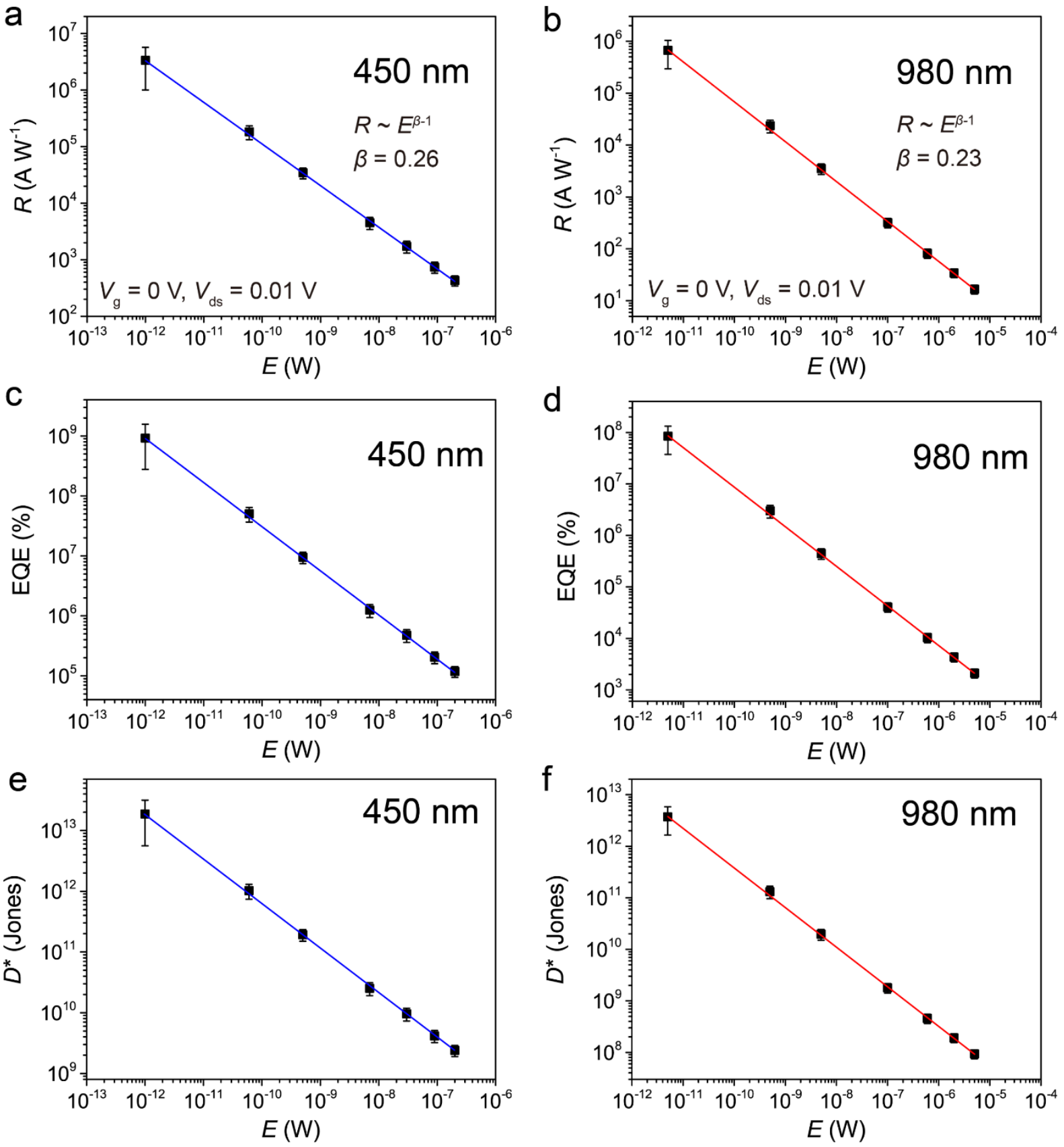

Figure S22. The responsivity $(R)$, EQE and specific detectivity $\left(D^{*}\right)$ as a function of effective light intensity for (a,c,e) $450 \mathrm{~nm}$ and (b,d,f) $980 \mathrm{~nm}$ light irradiation, respectively. $R$ and $E$ exhibit a relationship of $R \sim E^{\beta-1}$ with $\beta=0.26$ and 0.23 for $450 \mathrm{~nm}$ and $980 \mathrm{~nm}$ light irradiation, respectively. EQE and $D^{*}$ were calculated using equation of $\mathrm{EQE}=R \frac{h c}{\lambda e} \times 100 \%$ and $D^{*}=$ $R \sqrt{A} /{\sqrt{2 q I_{d}}}^{29} V_{\mathrm{g}}=0 \mathrm{~V}$ and $V_{\mathrm{ds}}=0.01 \mathrm{~V}$. 


\section{Supplementary Note 3: Spatial Distribution of Trapped Charges in Pyr-GDY and PbS QDs}

A theoretical model given by Queisser ${ }^{30}$ is used to analyze the decay dynamics of the persistent photoconductivity (PPC) caused by the trapped charges in Pyr-GDY and PbS QDs. Under light illumination, abundant electron-hole pairs are generated in Pyr-GDY or PbS QDs. Driven by the built-in electric field between graphene and Pyr-GDY (PbS QDs), the photogenerated electrons (holes) transfer to graphene while leaving holes (electrons) trapped in Pyr-GDY (PbS QDs). Here time $t=0$ denotes the moment when illumination is turned off, and $x=0$ represents the interface between graphene and Pyr-GDY or PbS QD film.

For the trapped holes in Pyr-GDY, it is assumed that the distribution follows the spatial Fermi hole distribution (Figure S23a), which dictates that holes preferentially occupy the trap sites near the Pyr-GDY/Gr interface. With the increase of the distance from the interface $(x>0)$, the decrease of the hole density in Pyr-GDY follows the equation of: ${ }^{30}$

$$
p_{0} \equiv p(x, t=0)=Z\left\{1+\exp \left[\left(x-x_{f}\right) / L\right]\right\}^{-1},
$$

where $Z$ represents the volume density of hole-capturing sites in Pyr-GDY $(x>0)$, and $x_{f}$ is the distance at which half of the trap sites are occupied by holes.

The excess number of electrons left behind in graphene is equal to the number of trapped holes in Pyr-GDY (Figure S24a), which can be expressed as: ${ }^{30}$

$$
\Delta n=\int_{0}^{d} p(x, t=0) d x \quad \text { at } t=0,
$$

where $d$ is the thickness of Pyr-GDY.

For $t>0$, recombination of photogenerated electrons and holes occurs, and the electronhole pairs that are spatially close would recombine first. Figure S23b displays the distribution of holes remaining in Pyr-GDY at $t_{2}>t_{1}>0$, which can be described by the equation:

$$
\frac{d p(x, t)}{d t}=-\frac{p(x, t)}{\tau_{0}} \exp \left(-\frac{2 x}{a}\right),
$$

where $a$ is the Bohr radius in graphene, and $\tau_{0}$ is the lifetime of electron-hole pair without spatial separation. Thus the excess number of electrons in graphene can be expressed as:

$$
\Delta n(t)=\int_{0}^{d} p(x, t=0) \exp \left[-\left(t / \tau_{0}\right) \exp (-2 x / a)\right] d x .
$$


The sharp-front approximation given by Queisser is used to simplify the equation. ${ }^{30}$ At a given time $t\left(t>>\tau_{0}\right)$ all trap sites located before a particular distance $x_{s}$ are assumed to be neutralized (Figure S24b). The front $x_{s}$ is defined by assuming that all carriers with lifetime $t+$ $\tau_{0}$ have recombined at time $t$, that is:

$$
\begin{gathered}
\tau_{0} \exp \left(2 x_{s} / a\right)=t+\tau_{0}, \\
x_{s}(t)=\frac{a}{2} \ln \left(\frac{t}{\tau_{0}}\right) .
\end{gathered}
$$

The integral in Eq. (S4) can be simplified as:

$$
\begin{aligned}
\Delta n(t) & =\int_{x_{s}}^{d} p(x, t=0) d x \\
& =Z d\left[1-(a / 2 d) \ln \left(t / \tau_{0}\right)\right]
\end{aligned}
$$

From the above equations we can conclude that the recombination of the electrons in graphene and holes trapped in Pyr-GDY would become rather slow for time $t>>\tau$, indicating a PPC effect (Figure S25a).

A similar spatial Fermi electron distribution for the trapped electrons in PbS QD film can be demonstrated, which will also cause a remarkable PPC effect (Figure S25 b).

With the increase of light intensity and illumination duration, the number of the trapped charges in Pyr-GDY or PbS film increases. As discussed above, more charges will be trapped by the trapping sites far away from the interface, which results in a slow decay for the photocurrent on termination of light irradiation. 

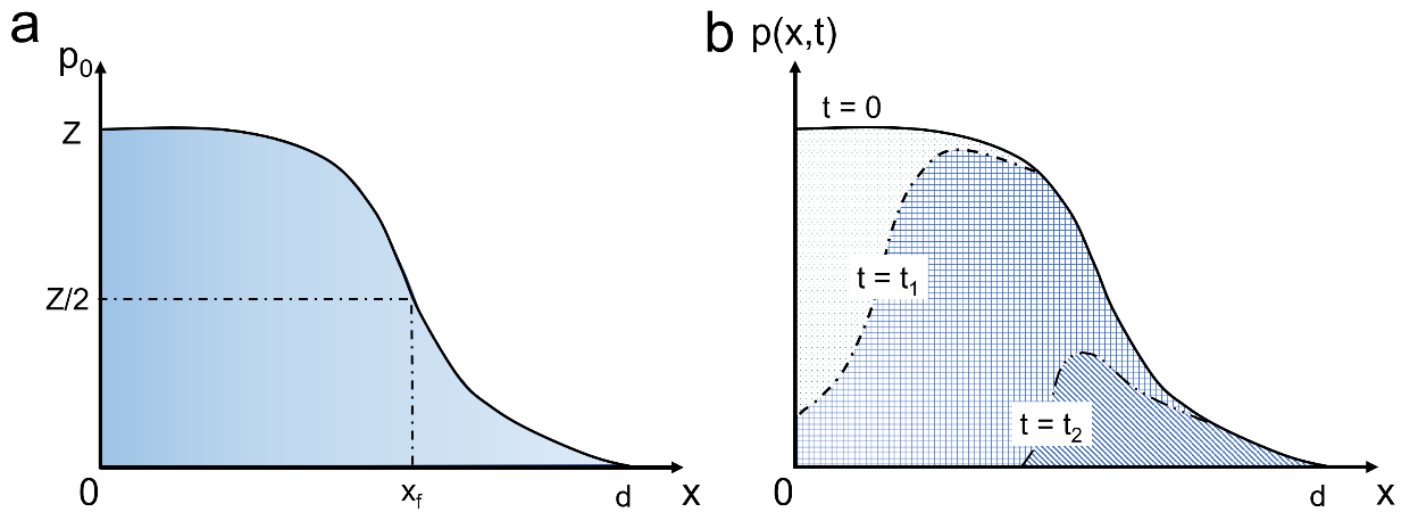

Figure S23. Spatial distribution of trapped charges in Pyr-GDY and PbS film. (a) Spatial distribution of trapped charges at $t=0$ on termination of irradiation. (b) Spatial distribution of trapped charges at time of $t_{1}$ and $t_{2}\left(t_{2}>t_{1}>0\right)$ after illumination.
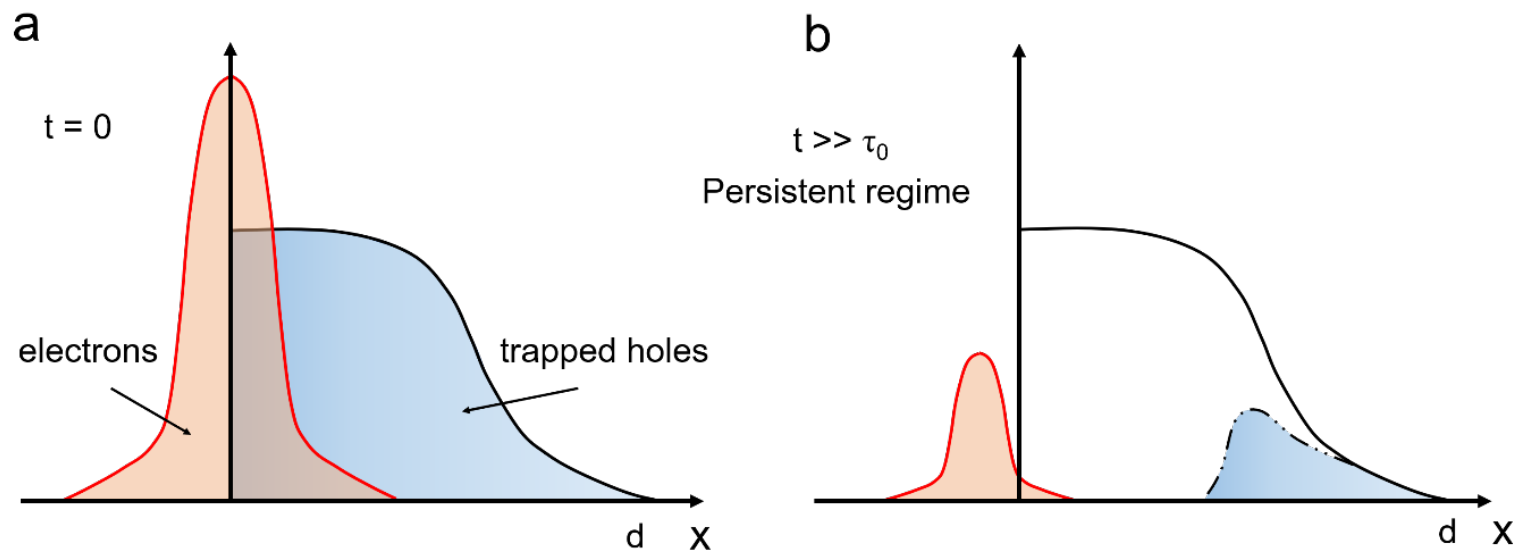

Figure S24. Evolution of the electron-hole distribution nearby the Gr/Pyr-GDY interface. (a) $t=0$ denotes the moment when light irradiation is turned off, and (b) $t>>\tau_{0}$ represents the time that is much longer than the lifetime of carriers. 

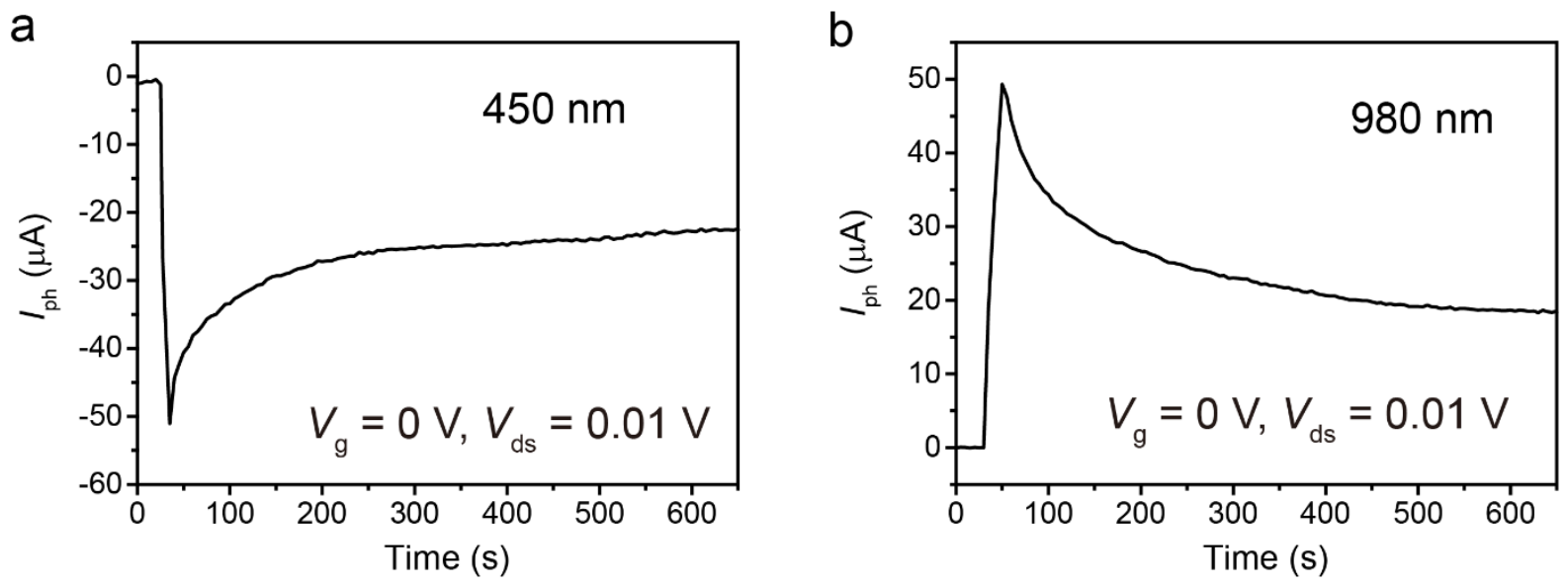

Figure S25. Dynamic photoresponse of the optical synapse under illumination of (a) $450 \mathrm{~nm}$ and (b) $980 \mathrm{~nm}$, respectively, exhibiting a negative/positive PPC effect. $V_{\mathrm{g}}=0 \mathrm{~V}$ and $V_{\mathrm{ds}}=0.01$ $\mathrm{V}$.
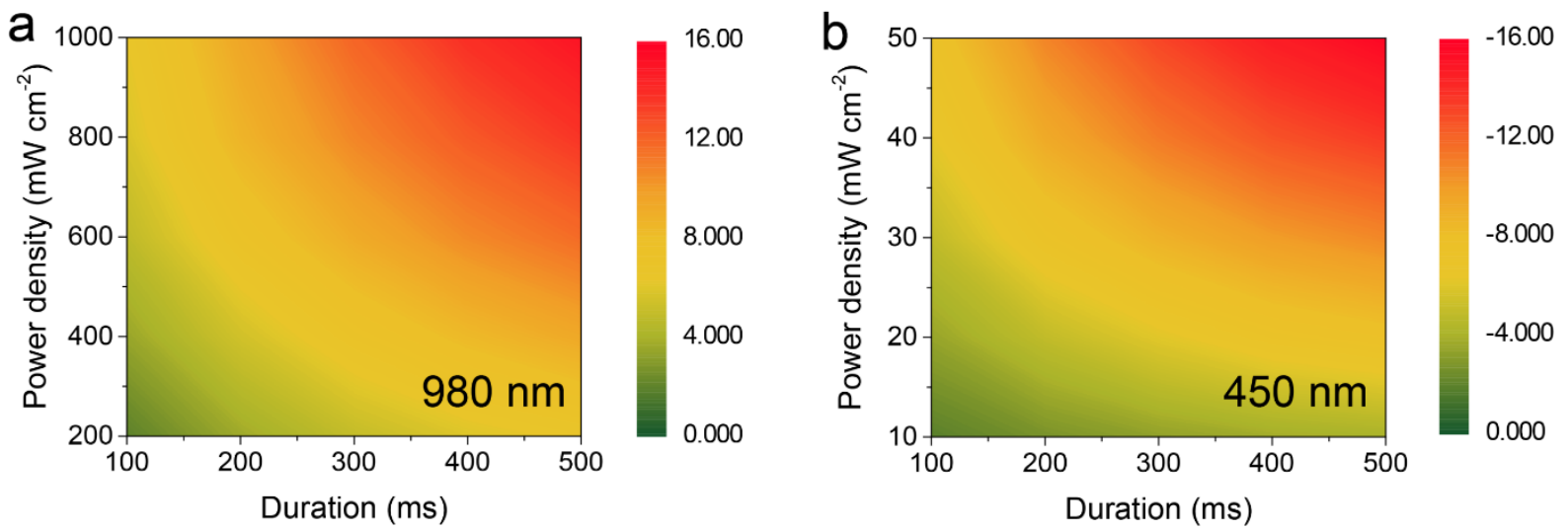

Figure S26. 2D color images of photocurrent as a function of illumination duration ( $x$-axis) and light intensity (y-axis) under light irradiation with wavelengths of (a) $980 \mathrm{~nm}$ and (b) 450 $\mathrm{nm}$, respectively.
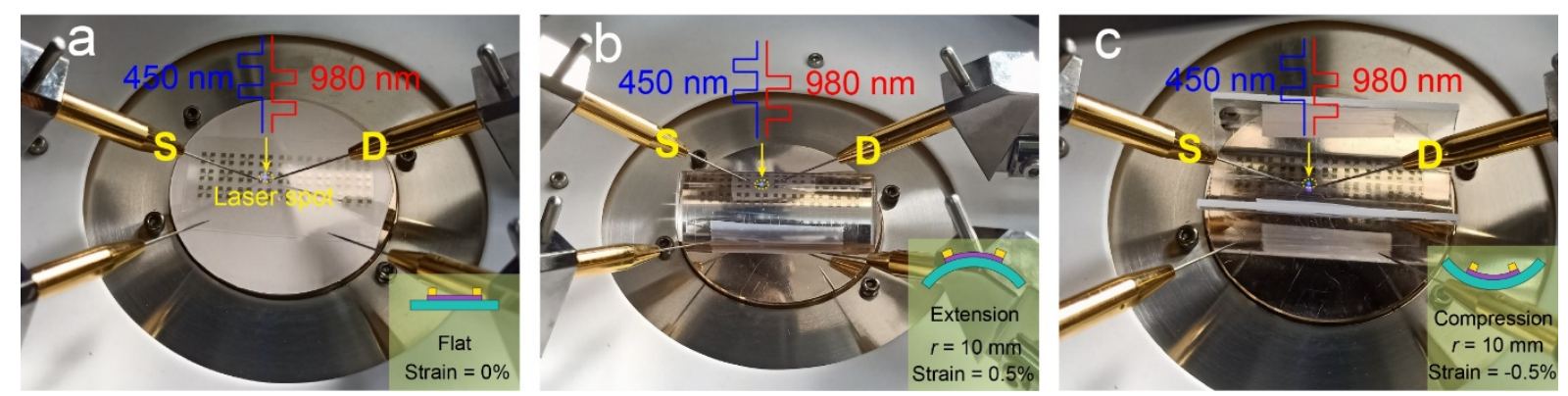

Figure S27. Measurement setup for the optical synapse at the (a) flat, (b) bending, and (c) folding states. The devices on PET film was attached to the outer and inner walls of a quartz tube with a radius of $10 \mathrm{~mm}$ in (b) and (c). The strain is extracted by $\operatorname{stain}=\frac{d}{2 r+d}$, where $d=$ $0.1 \mathrm{~mm}$ is the thickness of the PET film, and $r=10 \mathrm{~mm}$. 

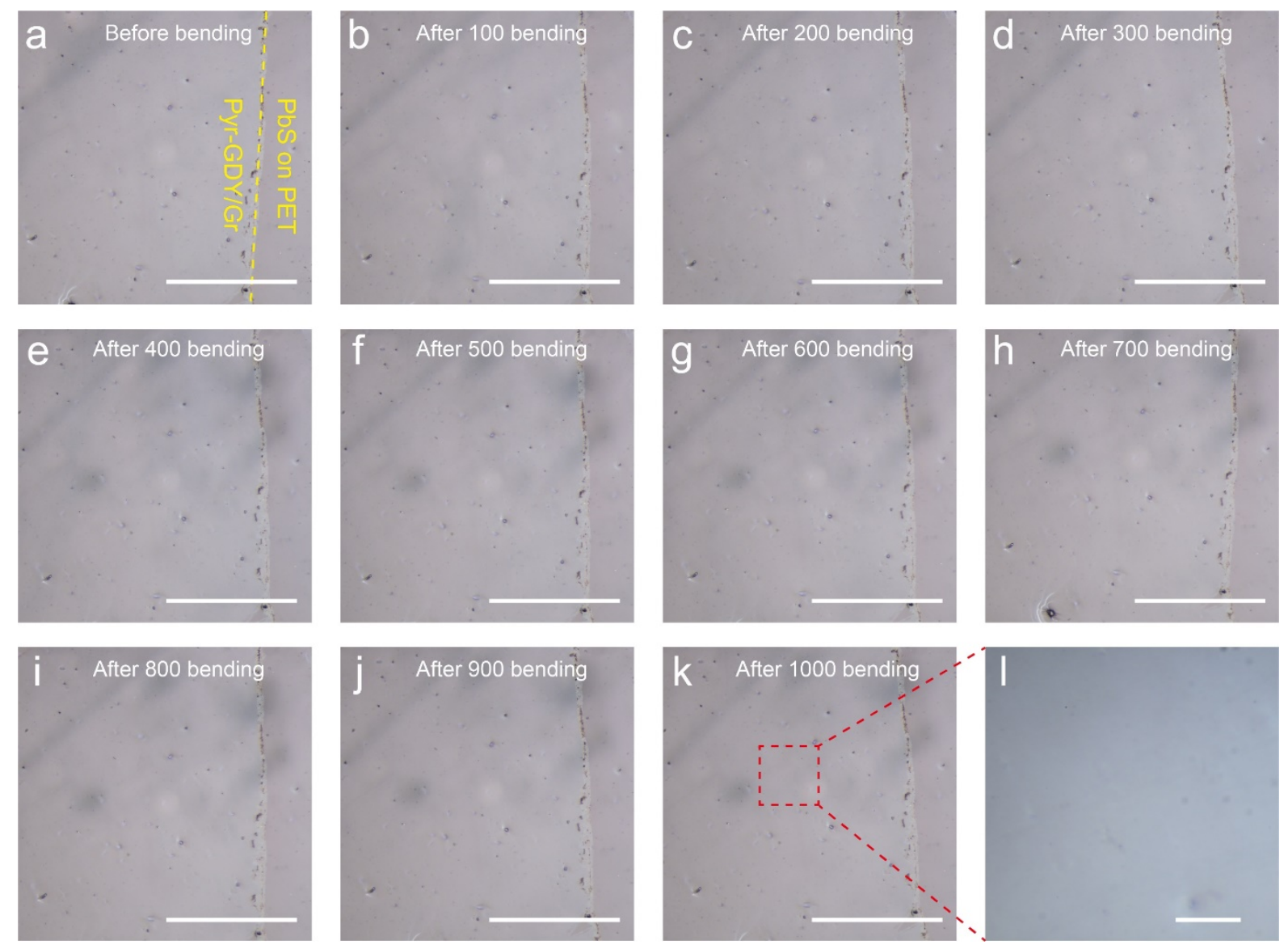

Figure S28. OM images of Pyr-GDY/Gr/PbS-QDs heterostructure on a flexible PET film (a) before and (b-l) after 100-1000 times bending cycles. Each bending cycle includes one extension and one compression of the film with a radius of $10 \mathrm{~mm}$. No obvious damage was observed even after 1000 bending, indicating the robust flexibility of the Pyr-GDY/Gr/PbSQDs heterostructure. Scale bars: $200 \mu \mathrm{m}$ in (a-k) and $20 \mu \mathrm{m}$ in (l). 


\section{Supplementary Note 4: Nonlinearity, Symmetricity and CCV analysis of the \\ LTP/LTD Curves}

\subsection{Nonlinearity}

The nonlinearity of the LTP/LTD curves was obtained by fitting the curves with the following weight update equations: 16,31,32

$$
\begin{aligned}
& G_{\mathrm{n}+1}=G_{\mathrm{n}}+\Delta G_{\mathrm{P}}=G_{\mathrm{n}}+\alpha_{\mathrm{p}} \exp \left(-\beta_{\mathrm{P}} \frac{G_{\mathrm{n}}-G_{\text {min }}}{G_{\text {max }}-G_{\text {min }}}\right) \text { (for LTP), } \\
& G_{\mathrm{n}+1}=G_{\mathrm{n}}+\Delta G_{\mathrm{D}}=G_{\mathrm{n}}-\alpha_{\mathrm{D}} \exp \left(-\beta_{\mathrm{D}} \frac{G_{\text {max }}-G_{\mathrm{n}}}{G_{\text {max }}-G_{\text {min }}}\right) \text { (for LTD), }
\end{aligned}
$$

where the $G_{n+1}$ and $G_{n}$ denote the synaptic conductance of the device when the $n+1^{\text {th }}$ and $n^{\text {th }}$ pulses are applied, and the parameters $\alpha$ and $\beta$ are the changing step sizes of the conductance and nonlinearity, respectively. As shown in Figure S29a, a larger $\beta$ presents a greater nonlinearity, and for an ideal synaptic device, its weight updating processes should be linear ( $\beta$ $=0$ ). The fitted parameters for LTP/LTD curves at flat and bending states are listed in Table S2. The fitted nonlinearity for the LTP and LTD curves of the optical synapse at flat state are 1.7 and 1.9, respectively (Figure S29b), demonstrating an outstanding linearity for pattern recognition.

\subsection{Symmetricity}

The symmetricity is defined as the reciprocal of the symmetric error, i.e., symmetricity = 1/symmetric error. ${ }^{32}$ The symmetric error can be calculated by the equation (Figure S30): ${ }^{32}$

$$
\begin{gathered}
\text { Symmetric error }=\sum_{k=1}^{k=n} \frac{\left(G_{N}(k)-G_{N}(2 n-k)\right)^{2}}{n}=\sum_{k=1}^{k=n} \frac{\left(\left(G(k)-G_{\min }\right)-\left(G(2 n-k)-G_{\min }\right)\right)^{2}}{n\left(G_{\max }-G_{\min }\right)^{2}} \\
=\sum_{k=1}^{k=n} \frac{(G(k)-G(2 n-k))^{2}}{n\left(G_{\max }-G_{\min }\right)^{2}}, \text { where } G_{N}(k)=\frac{G(k)-G_{\min }}{G_{\max }-G_{\min }} .
\end{gathered}
$$

Here $G_{N}$ represents the normalized conductance. The symmetric error calculated using Eq. (S10) is $\sim 0.12$, and thus the symmetricity is 8.42 for the LTP/LTD curves of the device at flat states. For the bending and folding states, the symmetricities are calculated to be 7.42 and 9.05 , respectively. 


\subsection{Cycle-to-cycle variation (CCV)}

The CCV, i.e. write noise, is defined as the standard deviation of the measured channel conductance during multiple cycles. ${ }^{13,33}$ As shown in Figure S31, the LTP/LTD cycles were performed for 15 times, and the maximum variations for LTP and LTD are 2.4\% and 2.8\%, respectively.
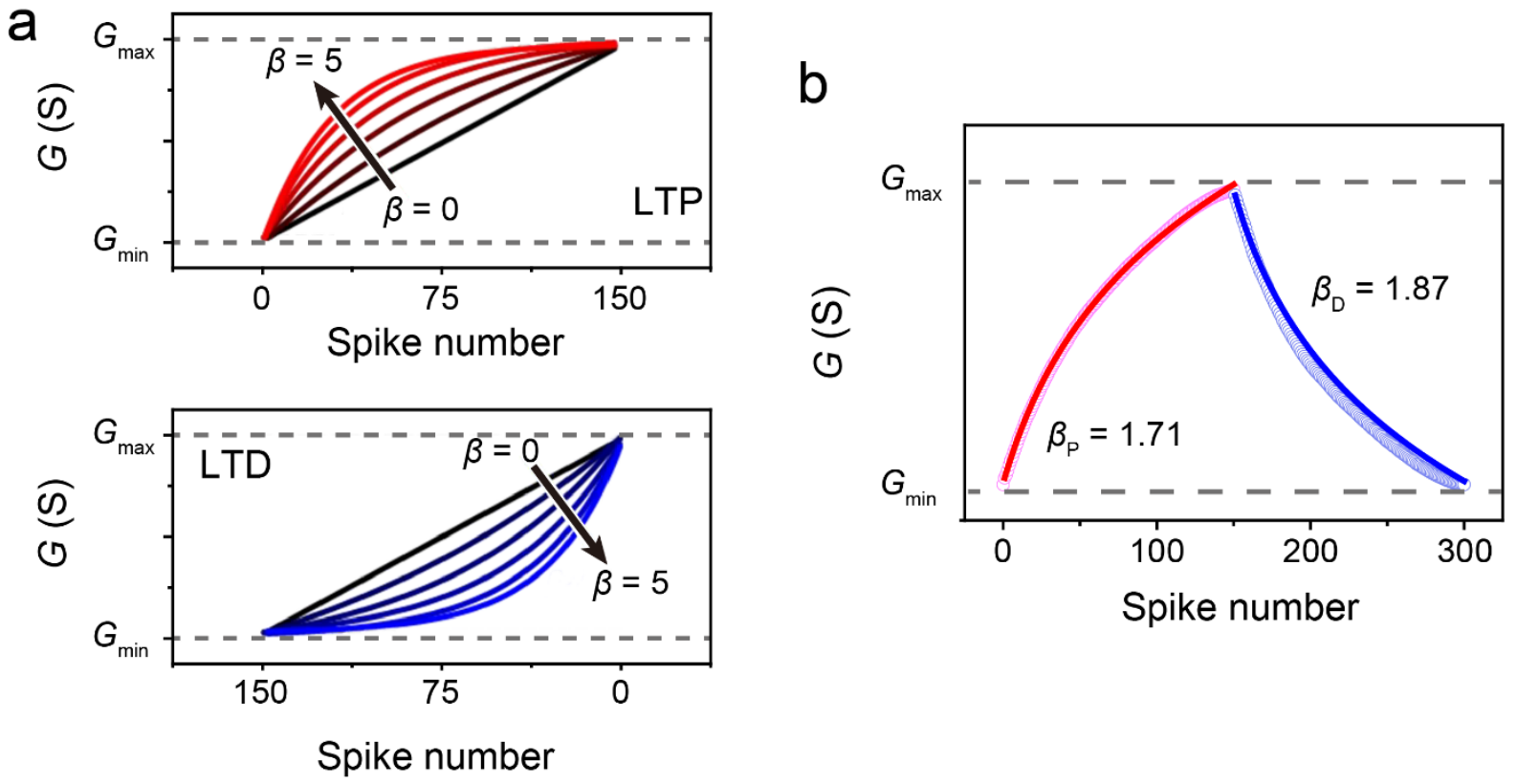

Figure S29. Nonlinearity analysis for LTP/LTD curves. (a) LTP and LTD with respect of the nonlinearity, $\beta$, ranging from 0 to 5 . (b) Fitting curves for LTP and LTD using the parameters given in Table $\mathrm{S} 2$. 


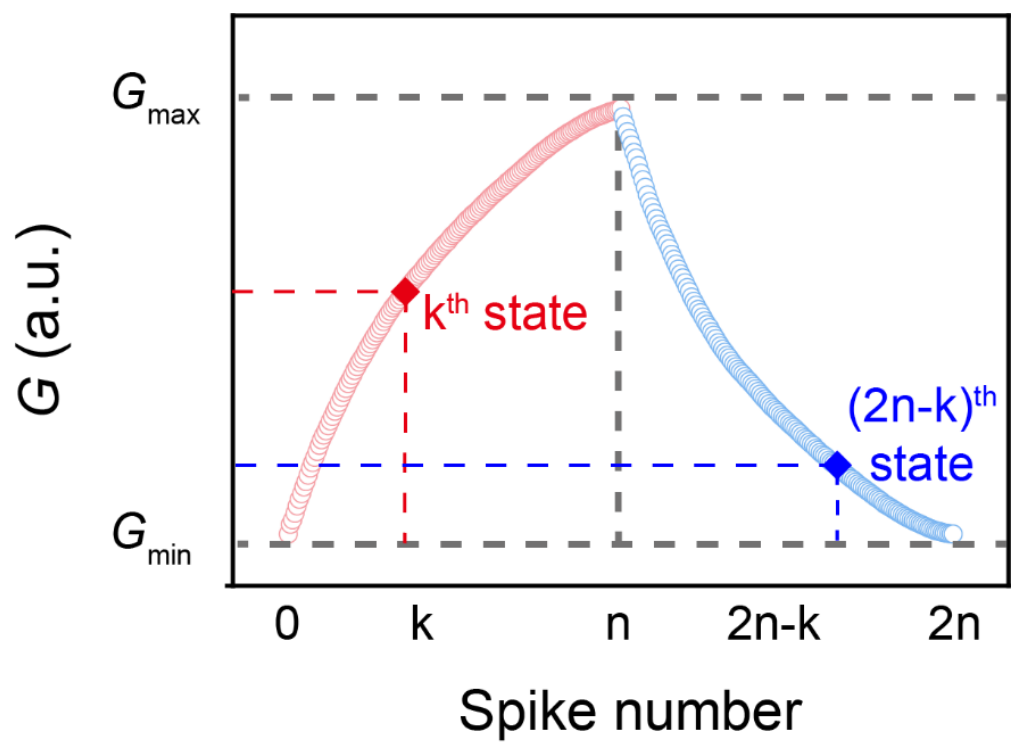

Figure S30. Symmetricity analysis for LTP/LTD curves.
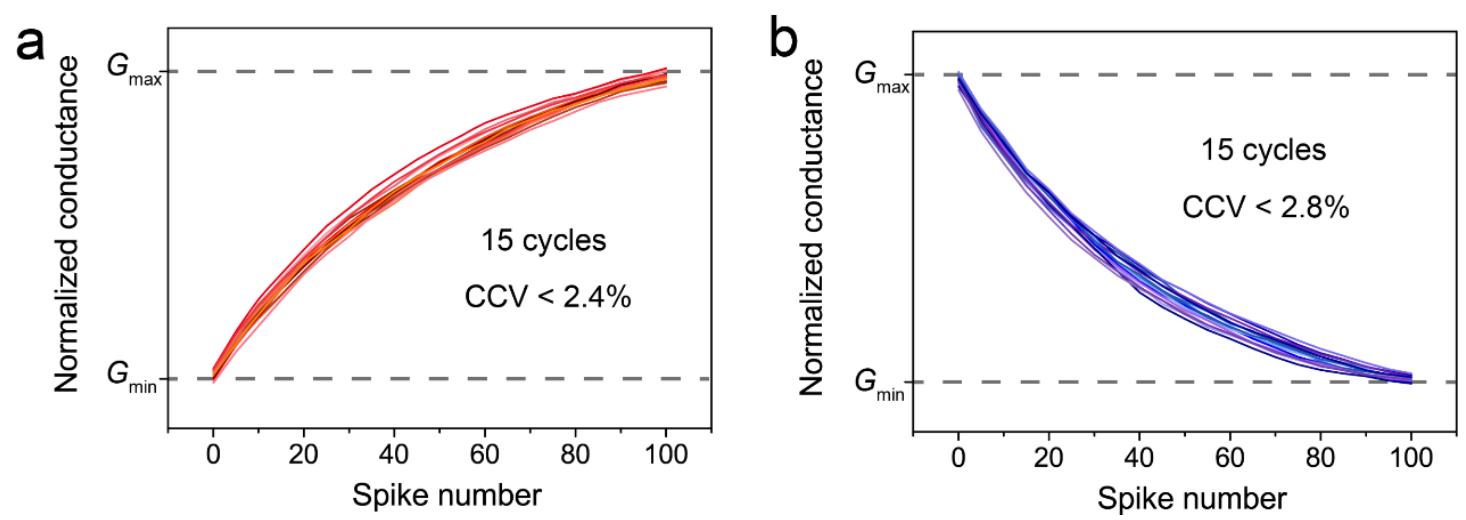

Figure S31. CCV analysis for (a) LTP and (b) LTD curves with 15 cycles.

Table S2. Extracted fitting parameters for the optical synapse at flat and bending states.

\begin{tabular}{c|cccccccc}
\hline State & $\alpha \mathrm{P}$ & $\alpha \mathrm{D}$ & $\beta_{\mathrm{P}}$ & $\beta_{\mathrm{D}}$ & $G_{\max }$ & $G_{\min }$ & Symmetricity & $\begin{array}{c}\text { Write } \\
\text { noise }\end{array}$ \\
\hline Flat & $2.8 \times 10^{-4} 3.3 \times 10^{-4}$ & 1.7 & 1.9 & $22.7 \mathrm{mS}$ & $7.3 \mathrm{mS}$ & 8.42 & $2.4 \% / 2.8 \%$ \\
\hline Bending & $2.6 \times 10^{-4} 3.2 \times 10^{-4}$ & 1.8 & 2.1 & $20.5 \mathrm{mS}$ & $7.0 \mathrm{mS}$ & 7.42 & $2.9 \% / 3.1 \%$ \\
\hline Folding & $2.5 \times 10^{-4} 2.8 \times 10^{-4}$ & 1.8 & 1.9 & $21.1 \mathrm{mS}$ & $7.1 \mathrm{mS}$ & 9.05 & $2.6 \% / 2.9 \%$ \\
\hline
\end{tabular}


Table S3. The A/D and D/A parameters for simulation.

\begin{tabular}{|c|c|c|}
\hline & Range & Bits \\
\hline Row Input & -1 to 1 & 8 \\
\hline Col Output & -6 to 6 & 8 \\
\hline Col Input & -1 to 1 & 8 \\
\hline Row Output & -4 to 4 & 7 \\
\hline Row Update & $-0.1 \alpha$ to $0.1 \alpha$ & 5 \\
\hline Col Update & -1 to 1 & 8 \\
\hline
\end{tabular}

$\alpha$ is the learning rate, which is chosen to be 0.1 in these simulations.

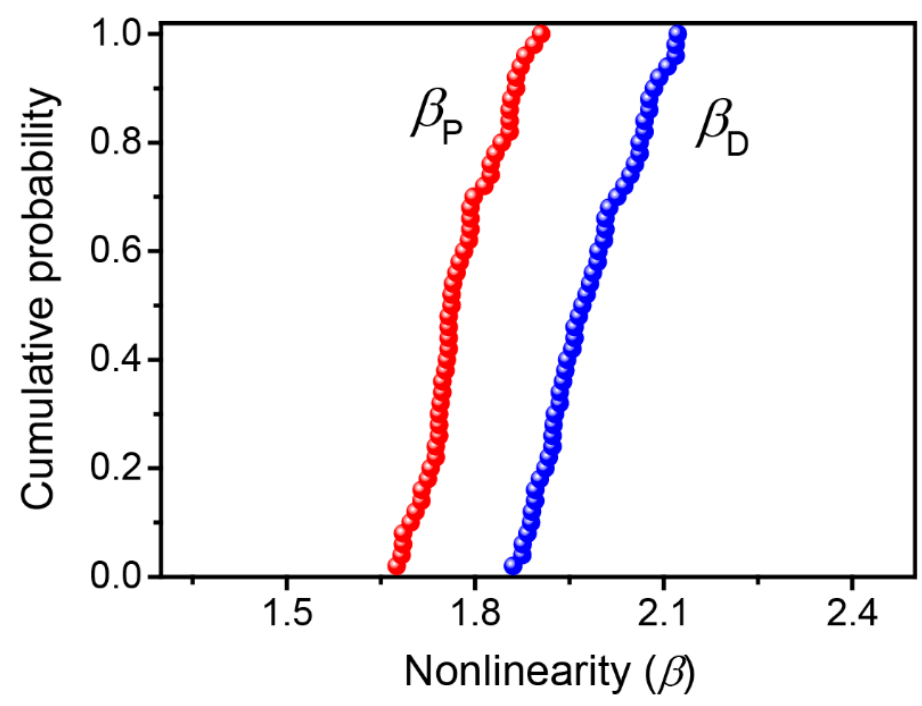

Figure S32. Device-to-device distribution of the nonlinearities for the LTP (red) and LTD (blue) curves, respectively. The statistical analysis is from the LTP/LTD curves of 50 devices fabricated by the same Pyr-GDY/Gr/PbS-QD heterostructure. 

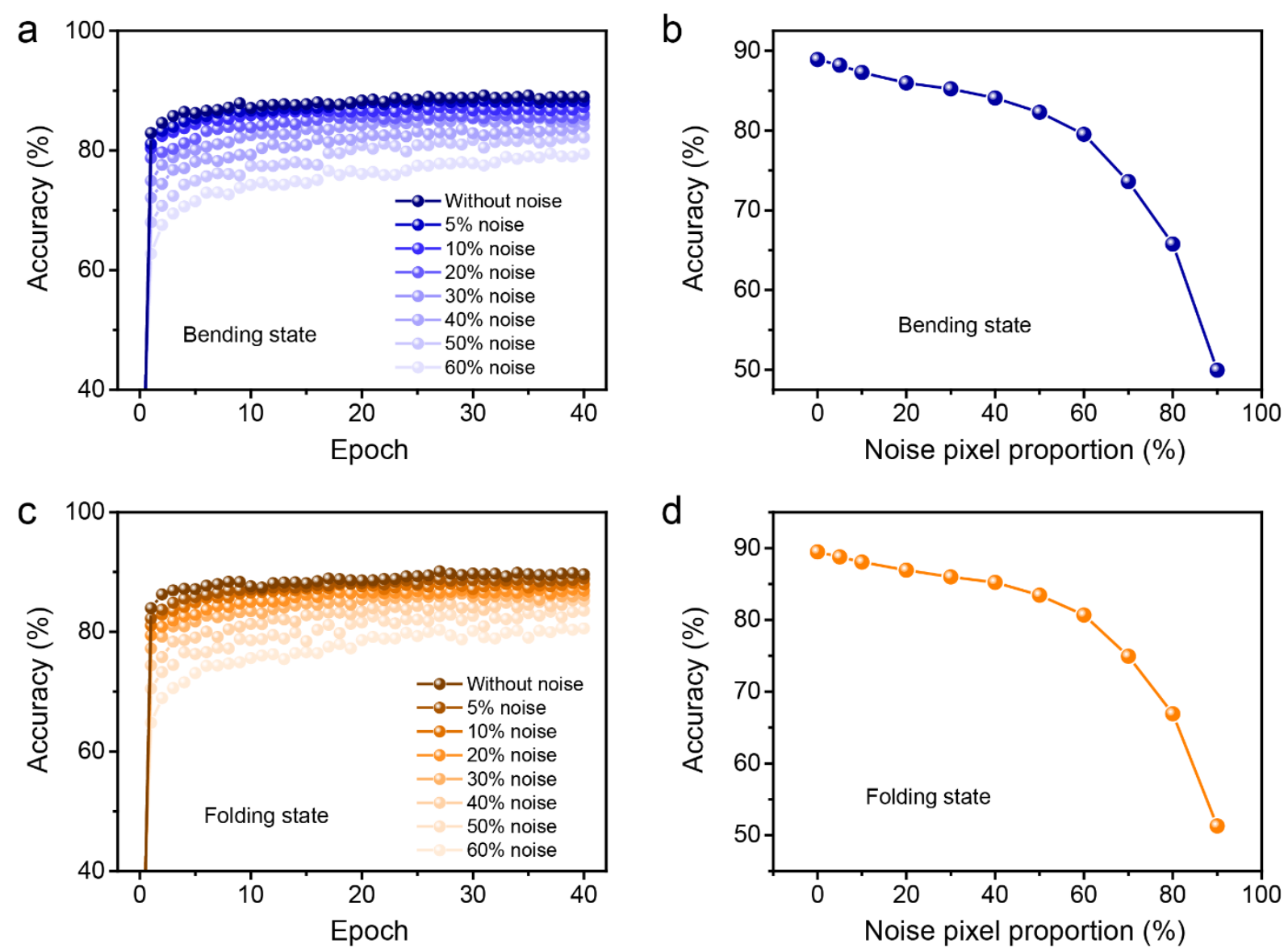

Figure S33. Image recognition for handwritten digits with different noise levels. (a,b) The recognition accuracy of the network in the bending state as a function of (a) training epochs and (b) noise pixel proportion. (c,d) The recognition accuracy of the network in the folding state as a function of (c) training epochs and (d) noise pixel proportion. 


\section{Supplementary Note 5: Linearly Modulated Conductance}

As illustrated in Figure S34, the conductance of graphene channel can be linearly modulated by the gate voltage around $V_{\mathrm{g}}=0 \mathrm{~V}$. The Pyr-GDY with trapped holes or PbS QDs with trapped electrons can be regarded as a positive or negative external gate for graphene channel, which will induce the shift of the Dirac voltage (Figure 1e in the main text). The change of the conductance $(\Delta G)$ and the trapped charges $(\Delta n)$ has a relationship of:

$$
\Delta G \propto \Delta I=A \cdot \Delta V=A \cdot \frac{e \cdot \Delta n}{C_{\mathrm{ox}}},
$$

where $A, e$ and $C_{\text {ox }}$ are the slope of the transfer curve around $V_{\mathrm{g}}=0 \mathrm{~V}$, elementary charge and capacitance per unit area, respectively. Therefore, the conductance of the graphene has a linearly relationship with the trapped charges. During the weight update processes, $\Delta n$ induced by each optical pulse is almost unchanged, resulting in a close to linear weight update trajectory.

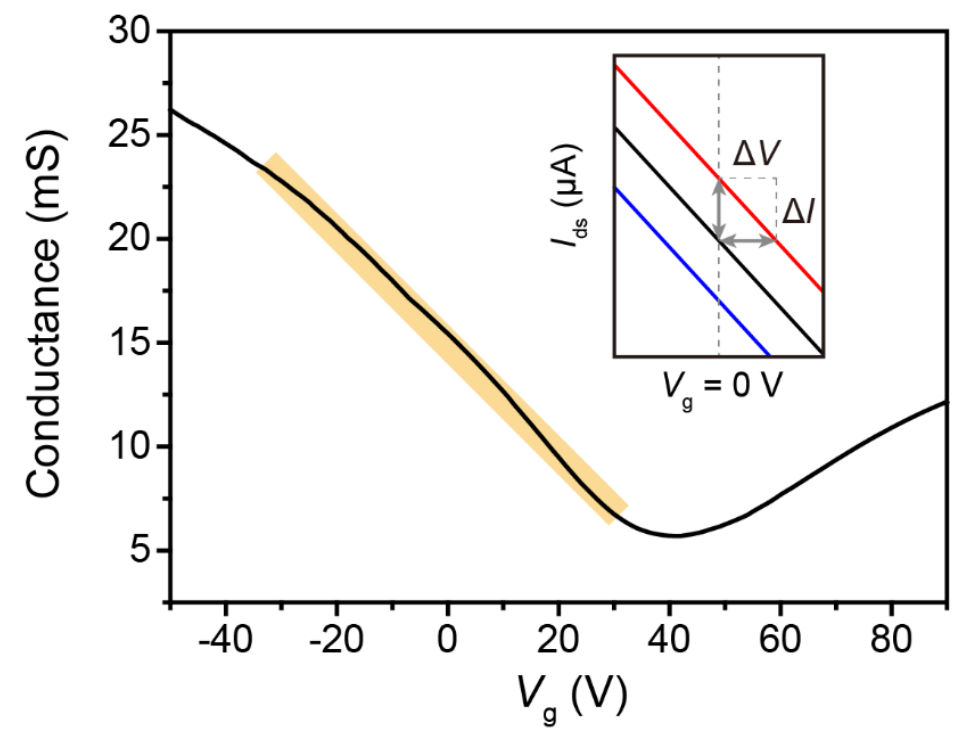

Figure S34. The transfer curve of the back-gated Pyr-GDY/Gr/PbS transistor. Inset illustrates the relationship between $\Delta I$ and $\Delta V$ around $V_{\mathrm{g}}=0 \mathrm{~V}$. 


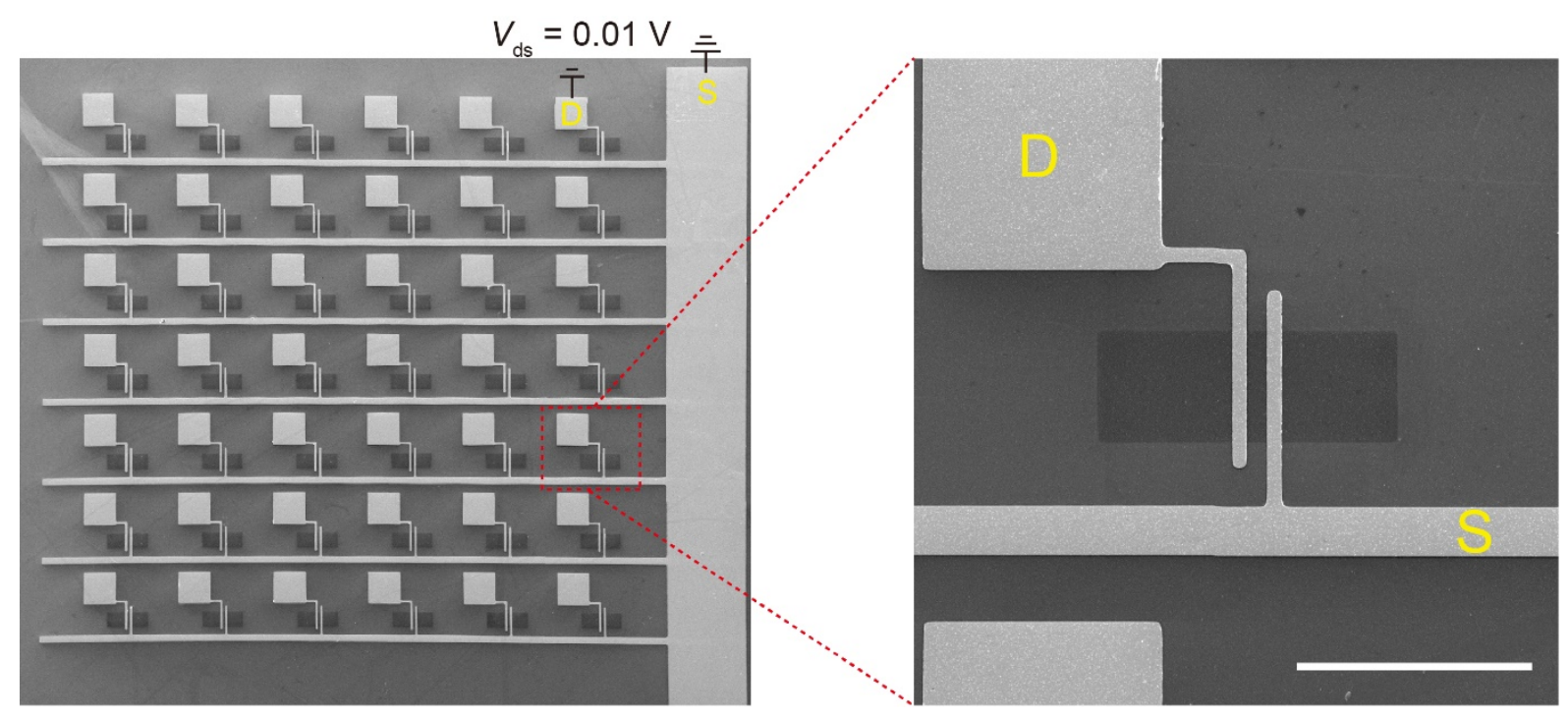

Figure S35. SEM images of the prepared $7 \times 6$ optical synapse array based on PyrGDY/Gr/PbS-QD heterostructure for visible information sensing-memory-processing system. Scale bar, $100 \mu \mathrm{m}$. Each synapse panel corresponds to one image pixel. Optical signals were applied one cell by one cell to input the letter images, followed by the current readout process with a readout voltage of $0.01 \mathrm{~V}$.

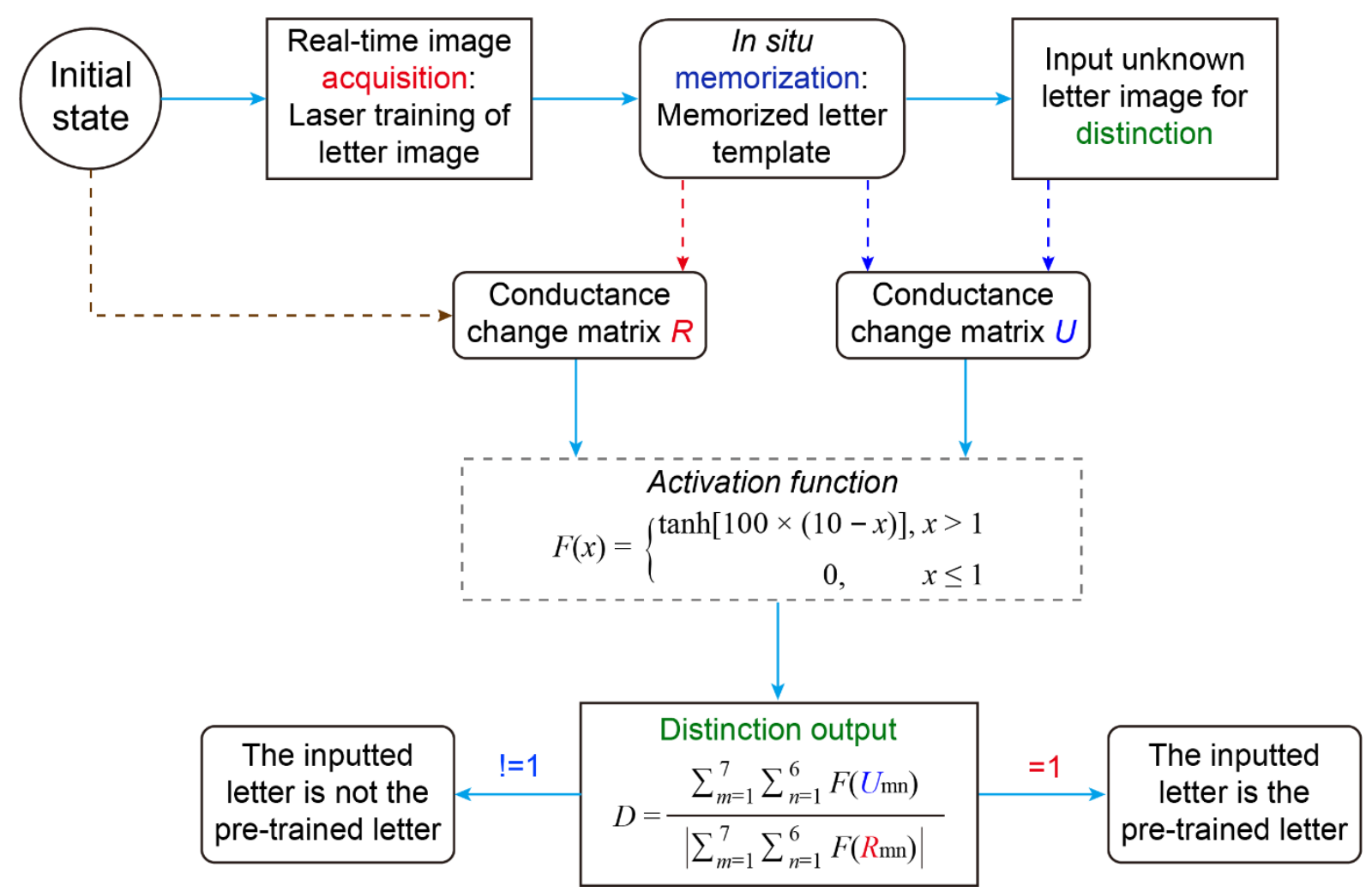

Figure S36. Process flow of the integrated visible information sensing-memory-processing system for real-time image detection, in situ image memorization and distinction tasks. 


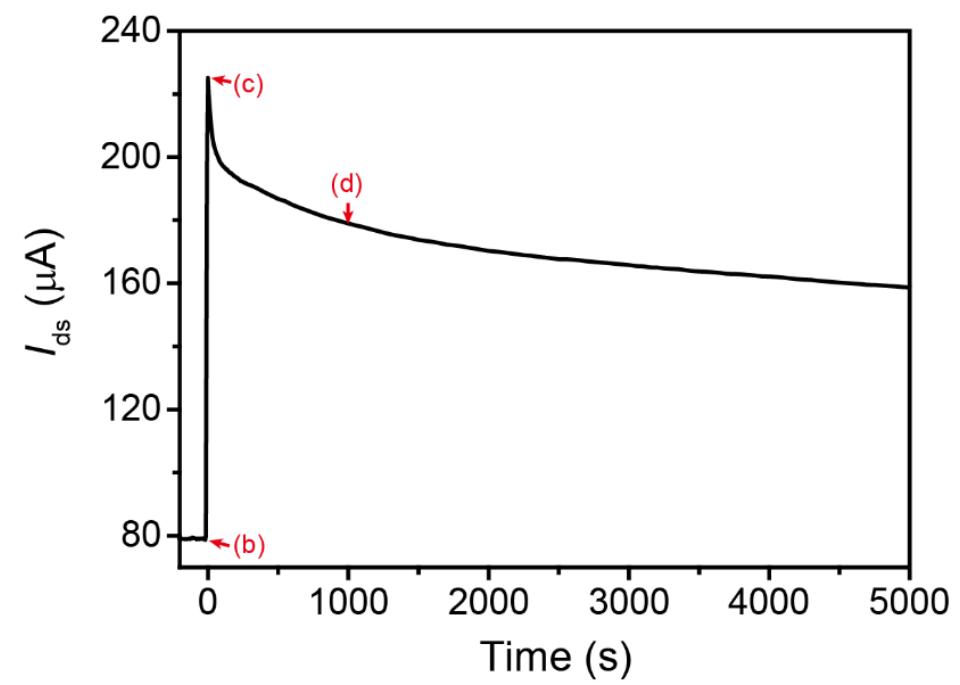

Figure S37. The retention characteristics of the Pyr-GDY/Gr/PbS-based device triggered by 150 successive optical spikes ( $980 \mathrm{~nm}, 150 \mathrm{~mW} \mathrm{~cm}^{-2}, 20 \mathrm{~ms}$ ). The marked points in the figure correspond to the conductance states as shown in Figure 5b,c,d, respectively.

$$
\begin{aligned}
& R=\left(\begin{array}{cccccc}
0.14 & 0.12 & 0.33 & 0.27 & 0.23 & 0.16 \\
0.12 & 0.26 & 15.42 & 15.3 & 0.17 & 0.32 \\
0.18 & 14.97 & 0.18 & 0.17 & 14.78 & 0.16 \\
0.17 & 15.63 & 0.09 & 0.14 & 15.41 & 0.19 \\
0.24 & 14.91 & 0.16 & 0.24 & 15.08 & 0.32 \\
0.23 & 0.25 & 15.12 & 14.49 & 0.31 & 0.09 \\
0.16 & 0.17 & 0.05 & 0.18 & 0.23 & 0.2
\end{array}\right) \\
& U_{1}=\left(\begin{array}{cccccc}
0.12 & 0.23 & 0.09 & 0.11 & 0.21 & 0.07 \\
0.09 & 0.18 & 6.89 & 6.39 & 15.14 & 0.17 \\
0.22 & 6.4 & 0.24 & 0.19 & 1.11 & 0.25 \\
0.24 & 6.55 & 15.18 & 14.5 & 6.91 & 0.19 \\
0.27 & 1.02 & 0.16 & 0.21 & 6.71 & 0.22 \\
0.17 & 14.42 & 6.69 & 7.54 & 0.16 & 0.08 \\
0.15 & 0.21 & 0.18 & 0.27 & 0.22 & 0.19
\end{array}\right) \\
& U_{2}=\left(\begin{array}{llllll}
0.25 & 0.05 & 0.18 & 0.16 & 0.19 & 0.12 \\
0.14 & 0.04 & 6.89 & 7.3 & 0.1 & 0.11 \\
0.25 & 6.94 & 0.11 & 0.15 & 6.59 & 0.13 \\
0.17 & 5.14 & 0.21 & 0.22 & 6.6 & 0.14 \\
0.14 & 6.67 & 0.08 & 0.1 & 6.97 & 0.16 \\
0.12 & 0.11 & 7.1 & 7.71 & 0.07 & 0.25 \\
0.16 & 0.17 & 0.13 & 0.21 & 0.1 & 0.2
\end{array}\right)
\end{aligned}
$$

Figure S38. The $7 \times 6$ matrixes $R, U_{1}$ and $U_{2}$ obtained by measuring the conductance of the 7 $\times 6$ optical synapse array before and after the input of the reference image and unknown images, respectively. 


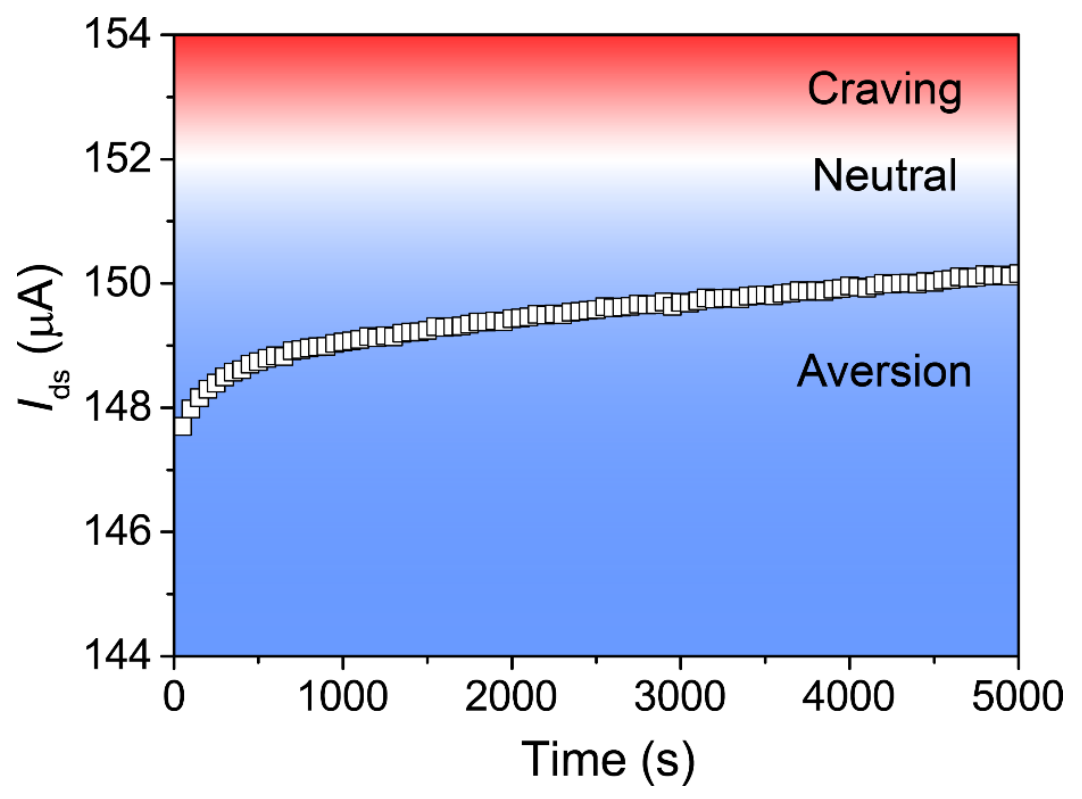

Figure S39. Retention time for the aversion state after treatment in the taste aversion learning. The channel current was measured every $50 \mathrm{~s}$ at $V_{\mathrm{g}}=0 \mathrm{~V}$ and $V_{\mathrm{ds}}=0.01 \mathrm{~V}$. 


\section{REFERENCES}

(1) Ahmed, T.; Tahir, M.; Low, M. X.; Ren, Y. Y.; Tawfik, S. A.; Mayes, E. L. H.; Kuriakose, S.; Nawaz, S.; Spencer, M. J. S.; Chen, H.; Bhaskaran, M.; Sriram, S.; Walia, S. Fully Light-Controlled Memory and Neuromorphic Computation in Layered Black Phosphorus. Adv. Mater. 2020, 2004207.

(2) Li, H.; Jiang, X.; Ye, W.; Zhang, H.; Zhou, L.; Zhang, F.; She, D.; Zhou, Y.; Han, S.-T. Fully Photon Modulated Heterostructure for Neuromorphic Computing. Nano Energy 2019, 65, 104000.

(3) Yang, C.-M.; Chen, T.-C.; Verma, D.; Li, L.-J.; Liu, B.; Chang, W.-H.; Lai, C.-S. Bidirectional All-Optical Synapses Based on a 2D Bi2 $\mathrm{O}_{2} \mathrm{Se} /$ Graphene Hybrid Structure for Multifunctional Optoelectronics. Adv. Funct. Mater. 2020, 30, 2001598.

(4) Zhou, F.; Zhou, Z.; Chen, J.; Choy, T. H.; Wang, J.; Zhang, N.; Lin, Z.; Yu, S.; Kang, J.; Wong, H. S. P.; Chai, Y. Optoelectronic Resistive Random Access Memory for Neuromorphic Vision Sensors. Nat. Nanotechnol. 2019, 14, 776-782.

(5) Gao, S.; Liu, G.; Yang, H.; Hu, C.; Chen, Q.; Gong, G.; Xue, W.; Yi, X.; Shang, J.; Li, R.-W. An Oxide Schottky Junction Artificial Optoelectronic Synapse. Acs Nano 2019, 13, 2634-2642.

(6) Zhu, X. J.; Lu, W. D. Optogenetics-Inspired Tunable Synaptic Functions in Memristors. Acs Nano 2018, 12, 1242-1249.

(7) Qin, S.; Wang, F.; Liu, Y.; Wan, Q.; Wang, X.; Xu, Y.; Shi, Y.; Wang, X.; Zhang, R. A Light-Stimulated Synaptic Device Based on Graphene Hybrid Phototransistor. 2D Mater. 2017, 4, 035022.

(8) Luo, Z.-D.; Xia, X.; Yang, M.-M.; Wilson, N. R.; Gruverman, A.; Alexe, M. Artificial Optoelectronic Synapses Based on Ferroelectric Field-Effect Enabled 2D Transition Metal Dichalcogenide Memristive Transistors. ACS Nano 2020, 14, 746-754.

(9) Hou, X.; Liu, C.; Ding, Y.; Liu, L.; Wang, S.; Zhou, P. A Logic-Memory Transistor with the Integration of Visible Information Sensing-Memory-Processing. Adv. Sci. 2020, 7, 2002072.

(10) Wang, S.; Chen, C.; Yu, Z.; He, Y.; Chen, X.; Wan, Q.; Shi, Y.; Zhang, D. W.; Zhou, H.; Wang, X.; Zhou, P. A MoS2/PTCDA Hybrid Heterojunction Synapse with Efficient Photoelectric Dual Modulation and Versatility. Adv. Mater. 2019, 31, 180622.

(11) Wang, T.-Y.; Meng, J.-L.; He, Z.-Y.; Chen, L.; Zhu, H.; Sun, Q.-Q.; Ding, S.-J.; Zhou, P.; Zhang, D. W. Ultralow Power Wearable Heterosynapse with Photoelectric Synergistic Modulation. Adv. Sci. 2020, 7, 1903480.

(12) Ni, Z.; Wang, Y.; Liu, L.; Zhao, S.; Xu, Y.; Pi, X.; Yang, D. Hybrid Structure of Silicon Nanocrystals and 2D WSe2 for Broadband Optoelectronic Synaptic Devices. 2018 IEEE International Electron Devices Meeting (IEDM). 2018, 38.35.31-38.35.34.

(13) Yin, L.; Han, C.; Zhang, Q.; Ni, Z.; Zhao, S.; Wang, K.; Li, D.; Xu, M.; Wu, H.; Pi, X.; Yang, D. Synaptic Silicon-Nanocrystal Phototransistors for Neuromorphic Computing. Nano Energy 2019, 63, 103859.

(14) Li, H. K.; Chen, T. P.; Liu, P.; Hu, S. G.; Liu, Y.; Zhang, Q.; Lee, P. S. A LightStimulated Synaptic Transistor with Synaptic Plasticity and Memory Functions Based on Ingaznox-Al2o3 Thin Film Structure. J. Appl. Phys. 2016, 119, 244505. 
(15) Wu, Q. T.; Wang, J. W.; Cao, J. C.; Lu, C. Y.; Yang, G. H.; Shi, X. W.; Chuai, X. C.; Gong, Y. X.; Su, Y.; Zhao, Y.; Lu, N. D.; Geng, D.; Wang, H.; Li, L.; Liu, M. Photoelectric Plasticity in Oxide Thin Film Transistors with Tunable Synaptic Functions. Adv. Electron. Mater. 2018, 4, 1800556.

(16) Sun, J.; Oh, S.; Choi, Y.; Seo, S.; Oh, M. J.; Lee, M.; Lee, W. B.; Yoo, P. J.; Cho, J. H.; Park, J.-H. Optoelectronic Synapse Based on IGZO-Alkylated Graphene Oxide Hybrid Structure. Adv. Funct. Mater. 2018, 28, 1804397.

(17) Duan, N.; Li, Y.; Chiang, H.-C.; Chen, J.; Pan, W.-Q.; Zhou, Y.-X.; Chien, Y.-C.; He, Y.-H.; Xue, K.-H.; Liu, G.; Chang, T.-C.; Miao, X.-S. An Electro-Photo-Sensitive Synaptic Transistor for Edge Neuromorphic Visual Systems. Nanoscale 2019, 11, 17590-17599.

(18) Qiu, W.; Huang, Y.; Kong, L.-A.; Chen, Y.; Liu, W.; Wang, Z.; Sun, J.; Wan, Q.; Cho, J. H.; Yang, J.; Gao, Y. Optoelectronic in-Ga-Zn-O Memtransistors for Artificial Vision System. Adv. Funct. Mater. 2020, 30, 2002325.

(19) Wang, K.; Dai, S. L.; Zhao, Y. W.; Wang, Y.; Liu, C.; Huang, J. Light-Stimulated Synaptic Transistors Fabricated by a Facile Solution Process Based on Inorganic Perovskite Quantum Dots and Organic Semiconductors. Small 2019, 15, 1900010.

(20) Park, H. L.; Kim, H.; Lim, D.; Zhou, H.; Kim, Y. H.; Lee, Y.; Park, S.; Lee, T. W. RetinaInspired Carbon Nitride-Based Photonic Synapses for Selective Detection of UV Light. Adv. Mater. 2020, 32, 1906899.

(21) Wang, Y.; Lv, Z. Y.; Chen, J. R.; Wang, Z. P.; Zhou, Y.; Zhou, L.; Chen, X. L.; Han, S. T. Photonic Synapses Based on Inorganic Perovskite Quantum Dots for Neuromorphic Computing. Adv. Mater. 2018, 30, 201802883.

(22) Sun, F. Q.; Lu, Q. F.; Liu, L.; Li, L. H.; Wang, Y. Y.; Hao, M. M.; Cao, Z. G.; Wang, Z. H.; Wang, S. Q.; Li, T.; Zhang, T. Bioinspired Flexible, Dual-Modulation Synaptic Transistors toward Artificial Visual Memory Systems. Adv. Mater. Technol. 2020, 5, 1900888.

(23) Dai, S.; Wu, X.; Liu, D.; Chu, Y.; Wang, K.; Yang, B.; Huang, J. Light-Stimulated Synaptic Devices Utilizing Interfacial Effect of Organic Field-Effect Transistors. ACS Appl. Mater. Inter. 2018, 10, 21472-21480.

(24) Xu, F.; Nishida, T.; Shinohara, K.; Peng, L.; Takezaki, M.; Kamada, T.; Akashi, H.; Nakamura, H.; Sugiyama, K.; Ohta, K.; Orita, A.; Otera, J. Trimethylsilyl Group Assisted Stimuli Response: Self-Assembly of 1,3,6,8Tetrakis((trimethysilyl)ethynyl)pyrene. Organometallics 2017, 36, 556-563.

(25) Gao, L.; Ren, W.; Xu, H.; Jin, L.; Wang, Z.; Ma, T.; Ma, L.-P.; Zhang, Z.; Fu, Q.; Peng, L.-M.; Bao, X.; Cheng, H.-M. Repeated Growth and Bubbling Transfer of Graphene with Millimetre-Size Single-Crystal Grains Using Platinum. Nat. Commun. 2012, 3, 699.

(26) Wang, X.-H.; Zhang, Z.-C.; Wang, J.-J.; Chen, X.-D.; Yao, B.-W.; Hou, Y.-X.; Yu, M.X.; Li, Y.; Lu, T.-B. Synthesis of Wafer-Scale Monolayer Pyrenyl Graphdiyne on Ultrathin Hexagonal Boron Nitride for Multibit Optoelectronic Memory. ACS Appl. Mater. Inter. 2020, 12, 33069-33075.

(27) Mattevi, C.; Kim, H.; Chhowalla, M. A Review of Chemical Vapour Deposition of Graphene on Copper. J. Mater. Chem. 2011, 21, 3324-3334. 
(28) Leong, W. S.; Wang, H.; Yeo, J.; Martin-Martinez, F. J.; Zubair, A.; Shen, P.-C.; Mao, Y.; Palacios, T.; Buehler, M. J.; Hong, J.-Y.; Kong, J. Paraffin-Enabled Graphene Transfer. Nat. Commun. 2019, 10, 867.

(29) Wu, H.; Si, H.; Zhang, Z.; Kang, Z.; Wu, P.; Zhou, L.; Zhang, S.; Zhang, Z.; Liao, Q.; Zhang, Y. All-Inorganic Perovskite Quantum Dot-Monolayer $\mathrm{MoS}_{2}$ MixedDimensional van der Waals Heterostructure for Ultrasensitive Photodetector. Adv. Sci. 2018, 5, 1801219.

(30) Queisser; Theodorou. Decay Kinetics of Persistent Photoconductivity in Semiconductors. Phys. Rev. B 1986, 33, 4027-4033.

(31) Seo, S.; Jo, S.-H.; Kim, S.; Shim, J.; Oh, S.; Kim, J.-H.; Heo, K.; Choi, J.-W.; Choi, C.; Oh, S.; Kuzum, D.; Wong, H. S. P.; Park, J.-H. Artificial Optic-Neural Synapse for Colored and Color-Mixed Pattern Recognition. Nat. Commun. 2018, 9, 5106.

(32) Seo, S.; Kang, B.-S.; Lee, J.-J.; Ryu, H.-J.; Kim, S.; Kim, H.; Oh, S.; Shim, J.; Heo, K.; Oh, S.; Park, J.-H. Artificial van der Waals Hybrid Synapse and Its Application to Acoustic Pattern Recognition. Nat. Commun. 2020, 11, 3936-3936.

(33) Yang, C.-S.; Shang, D.-S.; Liu, N.; Fuller, E. J.; Agrawal, S.; Talin, A. A.; Li, Y.-Q.; Shen, B.-G.; Sun, Y. All-Solid-State Synaptic Transistor with Ultralow Conductance for Neuromorphic Computing. Adv. Funct. Mater. 2018, 28, 1804170. 